\title{
A General Metriplectic Framework with Application to Dissipative Extended Magnetohydrodynamics
}

\author{
Baptiste Coquinot $^{1} \dagger$ and Philip J. Morrison ${ }^{2} \ddagger$ \\ 1'épartement de Physique, École Normale Supérieure, 24 rue Lhomond, 75005, Paris \\ ${ }^{2}$ Department of Physics and Institute for Fusion Studies, University of Texas at Austin, 2525 \\ Speedway, Austin, TX 78712
}

(Received xx; revised xx; accepted xx)

General equations for conservative yet dissipative (entropy producing) extended magnetohydrodynamics are derived from two-fluid theory. Keeping all terms generates unusual cross-effects, such as thermophoresis and a current viscosity that mixes with the usual velocity viscosity. While the Poisson bracket of the ideal version of this model have already been discovered, we determine its metriplectic counterpart that describes the dissipation. This is done using a new and general thermodynamic point of view for deriving dissipative brackets, a means of derivation that is natural for understanding and creating dissipative dynamics without appealing to underlying kinetic theory orderings. Finally the formalism is used to study dissipation in the Lagrangian variable picture where, in the context of extended magnetohydrodynamics, nonlocal dissipative brackets naturally emerge.

\section{Introduction}

\subsection{Background}

It is well known that the Hamiltonian dynamics of discrete and continuum systems may be written in terms of Poisson brackets (Landau \& Lifshitz (1960); Morrison (1998a)) with Hamiltonians. Such systems describe the evolution of a point in a phase space that may be finite-dimensional, the case for discrete systems, or infinite-dimensional, the case for continuum systems. The Poisson bracket $[f, g]$ is a bilinear operation on the set of smooth phase space functions $f, g$ or functionals (0-forms) that maps phase space to the real numbers. This set includes all the physical observables of interest. The Poisson bracket is also skew-symmetric, is a derivation, and satisfies the Jacobi identity; it generates the dynamics for any phase space function according to

$$
\frac{d f}{d t}=[f, \mathcal{H}],
$$

where the Hamiltonian $\mathcal{H}$, which is usually the energy, plays a special role. In the canonical case the Poisson bracket is nondegenerate and the Jacobi identity is equivalent to the associated symplectic 2-form being closed, while in the noncanonical case degeneracy gives rise to Casimir invariants, particular functionals having vanishing Poisson brackets with all functionals, i.e., Casimirs $C$ satisfy $[f, C]=0 \forall f$. The rich geometry of a phase space with a defined Poisson bracket, which includes symplectic and Poisson geometry, is intellectually very interesting and allows for better insight. Moreover, it is of practical value for understanding spectra, perturbation theory, and the construction of numerical

$\dagger$ Email address for correspondence: baptiste.coquinot@ens.fr

$\ddagger$ Email address for correspondence: morrison@physics.utexas.edu 
algorithms (see, e.g., Salmon (1983); Hagstrom \& Morrison (2011); Kraus et al. (2017); Morrison \& Vanneste (2016)).

Less known is the fact that dissipative dynamics can also emerge from brackets Kaufman \& Morrison 1982; Morrison 1984a; Kaufman 1984; Morrison 1984b; Grmela 1984; Morrison 1986) and the entropy $\mathcal{S}$ rather than the Hamiltonian $\mathcal{H}$ may serve as the generating function. (See Grmela \& Öttinger (1997a); Edwards (1998); Morrison (1998b); Kimura \& Morrison (2014); Materassi \& Morrison (2018); Gav-Balmaz \& Yoshimura (2017a, b); Eldred \& Gav-Balmaz (2018); Grmela \& Öttinger $(1997 b)$ for a selection of more recent theoretical work, and e.g. Kraus \& Hirvijoki (2017); Morrison (2017); Bressan et al. (2018) for recent numerical algorithms based on bracket dissipative structure.) Given that physical models generally contain both Hamiltonian and dissipative parts, we would like to use both kinds of brackets to get the complete dynamics. To this end we introduce a free energy $\mathcal{F}=\mathcal{H}-\mathcal{T S}$, where $\mathcal{T}$ is a Lagrange multiplier, interpreted as a generalized temperature (which is a uniform constant, in opposition to the physical temperature of the system $T$ ). This generalization is natural both because the free energy has a physical interpretation and because the entropy is a Casimir invariant of the Poisson bracket. The dynamics then becomes for any functional $f$ of the system,

$$
\frac{\mathrm{d} f}{\mathrm{~d} t}=[[f, \mathcal{F}]],
$$

where $[[f, g]]$ is an inclusive bracket, defined as the difference between a Poisson bracket, denoted $\{f, g\}$, and a dissipative bracket, denoted $(f, g)$. Since the energy is preserved and the entropy increases with time, $\mathcal{F}$ is a thermodynamic potential. Then, an equilibrium is given by $\delta \mathcal{F}=0$, where $\delta$ means the functional variation, which will be formally defined later.

An interesting property can already be proven. Upon denoting by $\sigma$ the entropy density, assuming that this variable appears in the Hamiltonian only through an internal energy density $u_{\mathrm{Vol}}$, and making use of the usual thermodynamic definition of the local temperature, $T=\partial u_{\mathrm{Vol}} / \partial \sigma$, we find the variation of $\mathcal{F}$ induced by a perturbation of the entropy $\delta \sigma$ gives $\delta \mathcal{F} / \delta \sigma=T-\mathcal{T}$. Therefore, at equilibrium, the temperature is uniform and equals the Lagrange multiplier $\mathcal{T}$, which validates our interpretation of this constant.

To be compatible with thermodynamics, there are requirements. The second law of thermodynamics is assured if the dissipative bracket has a nonnegative symmetrical bilinear form (we assume $\mathcal{T}$ to be nonnegative, which is consistent with its equilibrium interpretation). Then, it almost defines a metric. The first law requires the conservation of the Hamiltonian, i.e. $(\mathcal{H}, \mathcal{F})=0$. A stronger assumption is to require the degeneracy, i.e. $(\mathcal{H}, f)=0$ for any functional $f$. The situation is then symmetrical with the fact that $\mathcal{S}$ is a Casimir of the Poisson bracket. If such an assumption is fulfilled, the dissipative bracket is called metriplectic and metriplectic dynamics of any functional $f$ is given by

$$
\frac{\mathrm{d} f}{\mathrm{~d} t}=[[f, \mathcal{F}]]=\{f, \mathcal{H}\}+\mathcal{T}(f, \mathcal{S}) .
$$

The two principles of thermodynamics are then fulfilled by construction:

$$
\begin{aligned}
\frac{\mathrm{d} \mathcal{H}}{\mathrm{d} t} & =\{\mathcal{H}, \mathcal{H}\}+\mathcal{T}(\mathcal{H}, \mathcal{S})=0 \\
\frac{\mathrm{d} \mathcal{S}}{\mathrm{d} t} & =\{\mathcal{S}, \mathcal{H}\}+\mathcal{T}(\mathcal{S}, \mathcal{S})=\mathcal{T}(\mathcal{S}, \mathcal{S}) \geqslant 0 .
\end{aligned}
$$

The formalism above with the properties of symmetry and degeneracy first appeared in Morrison (1984a,$\underline{b})$, with the terminology metriplectic introduced in Morrison 
(1986). Several examples were given in these early works. Later it was called generic in Grmela \& Öttinger (1997a).

For a given physical system, it remains to determine the brackets. For fluid-like theories, the Poisson brackets naturally come from canonical brackets in terms of Lagrangian position and momentum variables, which can then be transformed into the usual Eulerian variables (see e.g. Morrison (1998a)). Poisson brackets for many models of plasma physics exist in the literature, including those for magnetohydrodynamics (Morrison \& Greene (1980); Morrison (2009)), relativistic magnetohydrodynamics D'Avignon et al. (2015) and extended magnetohydrodynamics (D'Avignon et al. (2016)). They are also known in other subjects of physics, e.g., in geophysical fluids (Bannon (2003)) and elasticity (Edwards \& Beris (1991)). Fewer metriplectic brackets are known; however, they have been discovered for fluids with viscosity and thermal diffusion (Morrison (1984b)), elasticity (Edwards \& Beris (1991)), and magnetohydrodynamics (Materassi \& Tassi (2012)). A comprehensive Lagrangian based approach, as opposed to our bracket approach, is given for $n$-fluid models with chemical reactions and general multicomponent fluids with irreversible processes in Eldred \& Gay-Balmaz (2018, 2020).

\subsection{Extended Magnetohydrodynamics: Model}

In this paper, we will mostly focus on extended magnetohydrodynamics. Extended magnetohydrodynamics may be derived from two-fluid theory, where ions and electrons are treated as distinct fluids. From this model, it is possible to get new equations in the usual variables, the center-of-mass velocity $\mathbf{v}$ and the electrical current $\mathbf{j}$ (Lüst (1959); Kampen \& Felderhof (1967)) (see also Lingam et al. (2016); Keramidas Charidakos et al. (2014)). To simplify the equations, two assumptions are made: quasineutrality, viz. that the densities of electrons and ions are assumed equal, and that the ratio of masses of electrons and ions, $\mu=m_{e} / m_{i}$, is small so that one can expand in powers of $\mu$. With these assumptions, one gets a generalized equation of motion for the velocity and a generalized Ohm's law. The model restricted to zeroth order in $\mu$ is called Hall magnetohydrodynamics (HMHD), while retaining first order terms produces what has been called extended magnetohydrodynamics (XMHD).

The equations of XMHD are expressed in terms of the variables $\left(\rho, \sigma, \sigma_{e}, \mathbf{v}, \mathbf{j}, \mathbf{E}, \mathbf{B}\right)$, which are respectively the mass density, the total entropy density, the electron entropy density, the center-of-mass velocity, the electrical current and the electric and magnetic fields. If our plasma is confined in a domain $\Omega$, the three Eulerian scalars are functions from $\Omega \times \mathbb{R} \longrightarrow \mathbb{R}$, while the four vector fields are functions from $\Omega \times \mathbb{R} \longrightarrow \mathrm{T} \Omega$, where $\mathrm{T} \Omega$ stands for the tangent bundle of the manifold $\Omega$ (here taken to be simple, e.g., a three torus). For simplicity, we define $\chi=m_{i} / e$, with $e$ being the charge of both electrons and ions, and choose units such that $\mu_{0}=\varepsilon_{0}=c=1$. The three scalar fields satisfy the conservation laws,

$$
\partial_{t} \rho+\nabla \cdot(\rho \mathbf{v})=0, \quad \partial_{t} \sigma+\nabla \cdot(\sigma \mathbf{v})=0, \quad \partial_{t} \sigma_{e}+\nabla \cdot\left(\sigma_{e} \mathbf{v}_{e}\right)=0
$$

where at order one in $\mu$, the electron velocity $\mathbf{v}_{e}=\mathbf{v}-(1-\mu) \chi \mathbf{j} / \rho$. The center of mass velocity satisfies the momentum conservation law,

$$
\frac{\mathrm{d} \mathbf{v}}{\mathrm{d} t}=\partial_{t} \mathbf{v}+\mathbf{v} \cdot \nabla \mathbf{v}=-\frac{1}{\rho} \nabla P+\frac{\mathbf{j}}{\rho} \times \mathbf{B}-\mu \chi^{2} \frac{\mathbf{j}}{\rho} \cdot \nabla\left(\frac{\mathbf{j}}{\rho}\right),
$$

where the use of $\mathrm{d} / \mathrm{d} t$ for the advective derivative should be clear from context, while its 
counterpart is the generalized Ohm's law,

$$
\begin{aligned}
\mathbf{E}+\mathbf{v} \times \mathbf{B} & =\chi \frac{\mathbf{j}}{\rho} \times \mathbf{B}-\frac{\chi}{\rho} \nabla P_{e} \\
& +\mu \frac{\chi^{2}}{\rho}\left[\frac{\partial \mathbf{j}}{\partial t}+\nabla \cdot(\mathbf{v} \otimes \mathbf{j}+\mathbf{j} \otimes \mathbf{v})\right]-\mu \chi^{3} \frac{\mathbf{j}}{\rho} \cdot \nabla\left(\frac{\mathbf{j}}{\rho}\right) .
\end{aligned}
$$

Finally, the electromagnetic variables are linked by the pre-Maxwell equations,

$$
\partial_{t} \mathbf{B}+\nabla \times \mathbf{E}=0, \quad \nabla \times \mathbf{B}=\mathbf{j}, \quad \nabla \cdot \mathbf{B}=0 .
$$

To close the system, one must specify the internal energies per unit mass for both ions, $u_{i}\left(\rho_{i}, \sigma_{i}\right)$, and electrons, $u_{e}\left(\rho_{e}, \sigma_{e}\right)$, where $\rho_{i} \approx(1-\mu) \rho, \rho_{e} \approx \mu \rho$ and $\sigma_{i}=\sigma-\sigma_{e}$. Then, the pressures are determined by

$$
P_{i}=\rho_{i}^{2} \frac{\partial u_{i}}{\partial \rho_{i}}, \quad P_{e}=\rho_{e}^{2} \frac{\partial u_{e}}{\partial \rho_{e}}, \quad \text { and } \quad P=P_{i}+P_{e} .
$$

One can also simplify the XMHD equations by eliminating the variables $\mathbf{j}$ and $\mathbf{E}$, which is indeed useful since the Ohm's law and Ampere's equation are not evolution equations but constraint equations. Then, the phase space should be a submanifold of the $\left(\rho, \sigma, \sigma_{e}, \mathbf{v}, \mathbf{j}, \mathbf{E}, \mathbf{B}\right)$ vector space. Thus, we are able to reduce these variables to get an easier phase space. To this end, it is useful to define a new variable, $\mathbf{B}^{*}$, that we will refer to here as the drifted magnetic field,

$$
\mathbf{B}^{*}:=\mathbf{B}+\mu \chi^{2} \nabla \times\left(\frac{\mathbf{j}}{\rho}\right)=\mathbf{B}+\mu \chi^{2} \nabla \times\left(\frac{1}{\rho} \nabla \times \mathbf{B}\right) .
$$

This variable first appeared in Lingam et al. (2015b). Physically, this drift comes from the difference of inertia between ions and electrons. While the velocity represents mostly the movement of ions, the frozen-in property of the magnetic field (Alfven (1950)) (also see e.g. Kampen \& Felderhof (1967)) is related to the dynamics of electrons. This creates a drift between the velocity and the magnetic flux, which is taken into account in this drifted magnetic field.

The Ohm's law and the pre-Maxwell's equations then reduce to (D'Avignon et al. $(2016))$

$$
\begin{array}{r}
\partial_{t} \mathbf{B}^{*}=\nabla \times\left[\mathbf{v} \times \mathbf{B}^{*}-\frac{1}{\rho}(\nabla \times \mathbf{B}) \times \mathbf{B}^{*}+\mu \frac{\chi}{\rho}(\nabla \times \mathbf{B}) \times(\nabla \times \mathbf{v})\right] \\
-\frac{\chi}{\rho^{2}}\left(\nabla P_{e} \times \nabla \rho\right) .
\end{array}
$$

\subsection{Extended Magnetohydrodynamics: Geometry}

After the reduction of removing $\mathbf{E}$ and $\mathbf{j}$, the phase variables can be chosen to be $\left(\rho, \sigma, \sigma_{e}, \mathbf{m}, \mathbf{B}^{*}\right)$, where $\mathbf{m}=\rho \mathbf{v}$ is the momentum density and $\mathbf{B}^{*}$ is constrained to be a divergence-free vector field. However, the divergence-free constraint will be fulfilled if it is initially true, because it turns out the dynamics will propagate it. Then, we define the local phase space at a point $\mathbf{x} \in \Omega$ as

$$
\Phi_{\mathbf{x}}=\mathbb{R}_{\rho} \times \mathbb{R}_{\sigma} \times \mathbb{R}_{\sigma_{e}} \times\left(\mathrm{T}_{\mathbf{x}} \Omega\right)_{\mathbf{m}} \times\left(\mathrm{T}_{\mathbf{x}} \Omega\right)_{\mathbf{B}^{*}}
$$

and the global phase space $\Phi$ as the sections of the bundle

$$
\amalg_{\mathbf{x} \in \Omega} \Phi_{\mathbf{x}} \longrightarrow \Omega .
$$


That is, a point of the global phase space gives an element of the local phase space for each spatial position, which describes uniquely the state of our system. We then define a functional as a map from $\Phi \longrightarrow \mathbb{R}$ (or $\mathbb{R}^{3}$ for vectors), a bracket as a bilinear operator $\mathbb{R}^{\Phi} \times \mathbb{R}^{\Phi} \longrightarrow \mathbb{R}^{\Phi}$ (where $\mathbb{R}^{\Phi}$ denotes the map from $\Phi$ to $\mathbb{R}$ ), that fulfills the Leibniz rule, the variation of a functional $f$ as $\delta f: \Phi \times \Phi \longrightarrow \mathbb{R}$ given by

$$
\delta f(\varphi, \delta \varphi)=\lim _{\epsilon \rightarrow 0} \frac{f(\varphi+\epsilon \delta \varphi)-f(\varphi)}{\epsilon}
$$

which can be viewed as directional derivative of $f$ at $\varphi$ in the direction $\delta \varphi$, and the functional derivative of $f$ at a point $\delta \varphi \in \Phi$, denoted $\delta f / \delta \varphi$, as the functional, when it exists, that satisfies,

$$
\delta f(\varphi, \delta \varphi)=\int_{\Omega} \frac{\delta f}{\delta \varphi} \delta \varphi
$$

for any $\varphi \in \Phi$, where the volume element $\left(e . g \cdot \mathrm{d}^{3} x\right)$ will not be stated when there is no likelihood of confusion. We consider a particular physical path in the phase space, parametrized by the time $\mathbb{R}$, and then functionals may be seen as functions of time. Let us also notice that a function on a local phase space $g: \Phi_{\mathbf{x}} \longrightarrow \mathbb{R}$, where $\mathbf{x} \in \Omega$, may be seen as a functional $g^{x}: \varphi \in \Phi \longrightarrow \int_{\Omega} g(\varphi(\mathbf{y})) \delta_{\Omega}(\mathbf{x}-\mathbf{y}) \mathrm{d}^{3} y$, where $\delta_{\Omega}$ is the Dirac distribution on $\Omega$ (and assuming that we can define $g$ over any local phase space, which is natural in practice). Without changing notations we identify $g$ with $g^{x}$.

Finally, one must define the important functionals $\mathcal{S}$ and $\mathcal{H}$. First, of course, we have the total entropy,

$$
\mathcal{S}=\int_{\Omega} \sigma
$$

Second, the energy per unit volume contains the kinetic energy, the internal energy, the magnetic energy, and also the kinetic energy of the electrons (Kimura \& Morrison (2014) $)$. We denote the global internal energy per unit mass $u=\left(\rho_{i} u_{i}+\rho_{e} u_{e}\right) / \rho=$ $(1-\mu) u_{i}+\mu u_{e}$. All together, this gives the total energy density,

$$
\varepsilon=\frac{1}{2} \rho|\mathbf{v}|^{2}+\rho u+\frac{1}{2}|\mathbf{B}|^{2}+\frac{1}{2} \mu \frac{\chi^{2}}{\rho}|\mathbf{j}|^{2}=\frac{|\mathbf{m}|^{2}}{2 \rho}+\rho u+\frac{1}{2} \mathbf{B} \cdot \mathbf{B}^{*},
$$

where use has been made of (1.1) and in the last equality a total divergence has been dropped. Thus this and other equalities involving integrands should be interpreted modulo a surface term. Then, the Hamiltonian is

$$
\mathcal{H}=\int_{\Omega} \varepsilon=\int_{\Omega}\left(\frac{|\mathbf{m}|^{2}}{2 \rho}+\rho u+\frac{1}{2} \mathbf{B} \cdot \mathbf{B}^{*}\right)
$$

For XMHD, the following Poisson bracket on e.g. functionals $f, g$, which was first given in Abdelhamid et al. (2015) based on the earlier work of Kimura \& Morrison (2014); Lingam et al. (2015b), together with the Hamiltonian of (1.4) produces the equations of 
motion:

$$
\begin{gathered}
\{f, g\}=\int_{\Omega} \mathrm{d}^{3} y\left[\rho \frac{\delta f}{\delta \mathbf{m}(\mathbf{y})} \cdot \nabla\left(\frac{\delta g}{\delta \rho(\mathbf{y})}\right)-\rho \frac{\delta g}{\delta \mathbf{m}(\mathbf{y})} \cdot \nabla\left(\frac{\delta f}{\delta \rho(\mathbf{y})}\right)\right. \\
+\sigma \frac{\delta f}{\delta \mathbf{m}(\mathbf{y})} \cdot \nabla\left(\frac{\delta g}{\delta \sigma(\mathbf{y})}\right)-\sigma \frac{\delta g}{\delta \mathbf{m}(\mathbf{y})} \cdot \nabla\left(\frac{\delta f}{\delta \sigma(\mathbf{y})}\right) \\
+\mathbf{m} \cdot\left(\frac{\delta f}{\delta \mathbf{m}(\mathbf{y})} \cdot \nabla\left(\frac{\delta g}{\delta \mathbf{m}(\mathbf{y})}\right)\right)-\mathbf{m} \cdot\left(\frac{\delta g}{\delta \mathbf{m}(\mathbf{y})} \cdot \nabla\left(\frac{\delta f}{\delta \mathbf{m}(\mathbf{y})}\right)\right) \\
+\mathbf{B}^{*} \cdot\left(\frac{\delta f}{\delta \mathbf{m}(\mathbf{y})} \cdot \nabla\left(\frac{\delta g}{\delta \mathbf{B}^{*}(\mathbf{y})}\right)\right)-\mathbf{B}^{*} \cdot\left(\frac{\delta g}{\delta \mathbf{m}(\mathbf{y})} \cdot \nabla\left(\frac{\delta f}{\delta \mathbf{B}^{*}(\mathbf{y})}\right)\right) \\
-\frac{\delta f}{\delta \mathbf{m}(\mathbf{y})} \cdot\left(\mathbf{B}^{*} \cdot \nabla\left(\frac{\delta g}{\delta \mathbf{B}^{*}(\mathbf{y})}\right)\right)+\frac{\delta g}{\delta \mathbf{m}(\mathbf{y})} \cdot\left(\mathbf{B}^{*} \cdot \nabla\left(\frac{\delta f}{\delta \mathbf{B}^{*}(\mathbf{y})}\right)\right) \\
-\frac{c \chi}{\rho}\left((1+\mu) \mathbf{B}^{*}-\mu \chi \nabla \times\left(\frac{\mathbf{m}}{\rho}\right)\right) \\
\left.\times\left(\nabla \times\left(\frac{\delta f}{\delta \mathbf{B}^{*}(\mathbf{y})}\right)\right) \times\left(\nabla \times\left(\frac{\delta g}{\delta \mathbf{B}^{*}(\mathbf{y})}\right)\right)\right]
\end{gathered}
$$

This bracket is clearly skew-symmetric in $f, g$ and it was shown by direct calculation in Abdelhamid et al. (2015) to satisfy the Jacobi identity. A much simplified proof of the Jacobi identity along with some remarkable connections to other models was obtained in Lingam et al. $(2015 \mathrm{a})$ and the bracket of (1.5) was derived from a Lagrangian variable action functional in D'Avignon et al. (2016).

Lastly, we note that strong boundary conditions are assumed such that all needed integrations by parts produce no boundary terms. In this paper, we will not consider any boundary effect on the brackets.

\subsection{Development - Overview}

Given the model and Hamiltonian structure of sections 1.2 and 1.3 respectively, it remains to discuss dissipation, the main content of our paper. The dynamical variables, the phase space, will remain the same, but the evolution equations will obtain new dissipative terms generated by a metriplectic bracket. A general form with several dissipative effects will be obtained by what amounts to a purely thermodynamic means. We emphasize that our approach differs from the very large plasma literature that yields fluid transport properties by appealing to particular kinetic theory orderings, e.g., the classic reference of Braginskii (1965) (see also (Kulsrud 1983)) and many subsequent highly detailed works.

In section 2 we start again from two-fluid theory, with general forms of thermal and viscous dissipative terms, to obtain dissipative XMHD. We will also consider cross-terms and look at their effect. For example, our model will include thermophoresis, and we will also discover a new current viscosity. This new viscosity will allow new cross effects between the velocity and magnetic field evolutions that may be of higher derivative orders while remaining linear.

In section 3 we will examine the dissipative brackets from a purely thermodynamic point of view, and find from this new perspective the brackets of hydrodynamics. Next we will introduce another set of variables that appear more natural for constructing the metriplectic bracket and then present a systematic way to derive general dissipative brackets for fluid-like systems.

In section 4 we will determine the complete brackets of dissipative XMHD. Keeping 
all cross effects, the dissipative bracket is given by equation (4.11) with the various phenomenological coefficients including cross effects described there.

In section [5] we discuss the Lagrangian variable picture of this model. While the Hamiltonian dynamics is well-known in terms of these variables, dissipation is usually considered only in the Eulerian picture. Consequently, we complete the picture by examining general forms of dissipation in the Lagrangian variable picture, and describe the transformation to the Eulerian picture as an example of metriplectic reduction.

Finally, in section 6 we conclude this work.

\section{From Two-Fluid Theory to Dissipative Extended Magnetohydrodynamics}

\subsection{Two-Fluid Theory}

In the two-fluid model, one considers the ions and the electrons as two distinct fluids with two velocity fields, $\mathbf{v}_{i}$ and $\mathbf{v}_{e}$, two mass densities, $\rho_{i}$ and $\rho_{e}$, and two pressures, $P_{i}$ and $P_{e}$. In addition one has the individual mass conservation laws,

$$
\partial_{t} \rho_{i}+\nabla \cdot\left(\rho_{i} \mathbf{v}_{i}\right)=0 \quad \text { and } \quad \partial_{t} \rho_{e}+\nabla \cdot\left(\rho_{e} \mathbf{v}_{e}\right)=0 .
$$

The quasineutrality assumption states, to first order in $\mu$,

$$
\rho_{i} \approx(1-\mu) \rho \quad \text { and } \quad \rho_{e} \approx \mu \rho,
$$

where $\rho=\rho_{i}+\rho_{e}$. The fluid equations for these variables are then coupled via the pre-Maxwell equations.

Conductivity arises from collisions between electrons and ions, and these are modeled by an exchange term in the momentum equation proportional to the relative velocity (see e.g. Kampen \& Felderhof (1967)). This term will express the resistivity of Ohm's law, with the bonus of a physical interpretation at this level. Plus, we know that in the onefluid theory, Ohm's law has a tensorial phenomenological constant, like what may occur for Fourier's law of heat conduction. From a thermodynamic point of view there could also be cross-terms between heat and electrical conduction (de Groot \& Mazur (1984)); consequently, for generality, we will add such cross terms in our two-fluid theory. To conserve the total momentum these appear with opposite signs.

A decision about temperature needs to be made. Although in many plasmas the electrons and ions have not relaxed, we will assume a common temperature. This is done for simplicity in this paper, mostly to not further complicate the presentation. This assumption allows us to drop the $\sigma_{e}$ variable, as will be discussed later, but generalizing the bracket by dropping this hypothesis is possible.

We assume that both fluids have their individual viscosities, which together will generate a one-fluid viscosity. This assumption naturally produces additional viscosities: a current viscosity and cross-effect viscosities, which do not appear to have been heretofore explored. Physically, all viscosities can be traced back to collisions between particles of the fluids. We are also able to add cross viscosity at the outset between the fluids, but this only alters the phenomenological coefficients that appear in our final theory. The terms we obtain can be interpreted as arising from collisions between ions and electrons of an higher order than the usual exchange terms.

Given the above assumptions, the two-fluid equations (cf. e.g. Kulsrud 1983) become

$$
\begin{gathered}
\rho_{i}\left(\partial_{t} \mathbf{v}_{i}+\mathbf{v}_{i} \cdot \nabla \mathbf{v}_{i}\right)=-\nabla P_{i}+\chi^{-1} \rho_{i}\left(\mathbf{E}+\mathbf{v}_{i} \times \mathbf{B}\right)-\frac{\rho^{2}}{\chi^{2}} \eta_{j j}\left(\mathbf{v}_{i}-\mathbf{v}_{e}\right) \\
-\frac{\rho}{\chi} \eta_{j T} \nabla T+\nabla \cdot\left[\Lambda_{i i} \nabla \mathbf{v}_{i}+\Lambda_{i e} \nabla \mathbf{v}_{e}\right]
\end{gathered}
$$


and

$$
\begin{gathered}
\rho_{e}\left(\partial_{t} \mathbf{v}_{e}+\mathbf{v}_{e} \cdot \nabla \mathbf{v}_{e}\right)=-\nabla \\
P_{e}-\chi^{-1} \rho_{i}\left(\mathbf{E}+\mathbf{v}_{e} \times \mathbf{B}\right)+\frac{\rho^{2}}{\chi^{2}} \eta_{j j}\left(\mathbf{v}_{i}-\mathbf{v}_{e}\right) \\
+\frac{\rho}{\chi} \eta_{j T} \nabla T+\nabla \cdot\left[\Lambda_{e i} \nabla \mathbf{v}_{i}+\Lambda_{e e} \nabla \mathbf{v}_{e}\right]
\end{gathered}
$$

where we have introduce six phenomenological coefficients. Two conductivities, electrical with $\eta_{j j}$ and thermic with $\eta_{j T}$, and four viscosity coefficients, for ions $\Lambda_{i i}$, electrons $\Lambda_{e e}$, and cross effects $\Lambda_{i e}$ and $\Lambda_{i e}$, where the latter are symmetric because of the Onsager relations. The conductivities are in general 2-tensors, i.e., matrices on $\mathrm{T}_{\mathbf{x}} \Omega$ at each point $\mathbf{x} \in \Omega$, whereas the viscosities are 4-tensors, i.e., matrices on the vector space of matrices on $\mathrm{T}_{\mathbf{x}} \Omega$. Thus, e.g., the bth-component of the term $\left.\nabla \cdot\left[\Lambda_{i i} \nabla \mathbf{v}_{i}\right]\right)$ in cartesian tensor notation, where repeated indices are summed, is $\partial_{a}\left[\left(\Lambda_{i i}\right)_{a b c d} \partial_{c}\left(v_{i}\right)_{d}\right]$, with $a, b, c, d \in$ $\{1,2,3\}$. Other tensor expressions here and henceforth should be interpreted similarly. We choose some constants with these coefficients, which change nothing since one may define the phenomenological coefficients another way, but will simplify the calculations.

For generality we allow the phenomenological coefficients to be arbitrary: they may be general tensors that depend on the phase space variables and position.

Lastly, thermodynamics gives two equations, the thermodynamic identities,

$$
T \mathrm{~d} s_{i}=\mathrm{d} u_{i}-\frac{P_{i}}{\rho_{i}^{2}} \mathrm{~d} \rho_{i} \quad \text { and } \quad T \mathrm{~d} s_{e}=\mathrm{d} u_{e}-\frac{P_{e}}{\rho_{e}^{2}} \mathrm{~d} \rho_{e},
$$

where $s_{i}$ and $s_{e}$ are the specific entropies of ions and electrons, while $u_{i}$ and $u_{e}$ are the specific internal energies of ions and electrons. Later, we will prefer to use the densities, which are related to the specific entropies and internal energies according to $\sigma_{\alpha}=\rho_{\alpha} s_{\alpha}$ and $u_{\alpha, \mathrm{Vol}}=\rho_{\alpha} u_{\alpha}$ for $\alpha \in\{i, e\}$.

\subsection{Toward One-Fluid Theory}

Next we define the center-of-mass velocity $\mathbf{v}$, the electrical current $\mathbf{j}$, and the total pressure $P$ by

$$
\mathbf{v}=\left(\rho_{i} \mathbf{v}_{i}+\rho_{e} \mathbf{v}_{e}\right) / \rho=\mathbf{v}_{i}+\frac{\mu}{1+\mu}\left(\mathbf{v}_{e}-\mathbf{v}_{i}\right), \quad \mathbf{j}=\rho_{i}\left(\mathbf{v}_{i}-\mathbf{v}_{e}\right) / \chi, \quad P=P_{i}+P_{e} .
$$

We may also write,

$$
\mathbf{v}_{i} \approx \mathbf{v}+\mu \chi \mathbf{j} / \rho \text { and } \mathbf{v}_{e} \approx \mathbf{v}-\chi \mathbf{j} / \rho
$$

Given the above change of variables and the quasineutrality assumption, equations (2.1) imply the one-fluid mass conservation law,

$$
\partial_{t} \rho+\nabla \cdot(\rho \mathbf{v})=0 .
$$

The one-fluid velocity equation and Ohm's law follow from the sum and difference of equations (2.2) and (2.3). The more difficult part of this computation is to manage the nonlinear terms. However, these terms are purely dynamical and not dissipative, and they have already been derived. For the detailed computation of these nonlinear terms, see Lüst (1959); D'Avignon et al. (2016). Summing equations (2.2) and (2.3) gives

$$
\begin{aligned}
\rho\left[\partial_{t} \mathbf{v}+\mathbf{v} \cdot \nabla \mathbf{v}+\mu \chi^{2} \frac{\mathbf{j}}{\rho} \cdot \nabla\left(\frac{\mathbf{j}}{\rho}\right)\right] & =\mathbf{j} \times \mathbf{B}-\nabla P \\
& +\nabla \cdot\left[\Lambda_{v v} \nabla \mathbf{v}+\Lambda_{v j} \nabla\left(\frac{\mathbf{j}}{\rho}\right)\right]
\end{aligned}
$$

where

$$
\Lambda_{v v}=\Lambda_{i i}+\Lambda_{i e}+\Lambda_{e i}+\Lambda_{e e}
$$


and

$$
\Lambda_{v j}=-\chi\left(\Lambda_{i e}+\Lambda_{e e}\right)+\mu \chi\left(\Lambda_{i i}+\Lambda_{e i}\right) .
$$

We can also define the viscosity 2 -tensor

$$
\Pi_{v}=\Lambda_{v v} \nabla \mathbf{v}+\Lambda_{v j} \nabla\left(\frac{\mathbf{j}}{\rho}\right) .
$$

The independent combination $\mu(1-\mu) \frac{\chi}{\rho} \times(2.2)-(1-\mu) \frac{\chi}{\rho} \times(2.3)$ gives the following:

$$
\begin{aligned}
\mu \frac{\chi^{2}}{\rho}\left[\frac{\partial \mathbf{j}}{\partial t}\right. & \left.+\nabla \cdot(\mathbf{v} \otimes \mathbf{j}+\mathbf{j} \otimes \mathbf{v})-\chi \mathbf{j} \cdot \nabla\left(\frac{\mathbf{j}}{\rho}\right)\right]+\eta_{j j} \mathbf{j}+\eta_{j T} \nabla T \\
& =\mathbf{E}+\left(\mathbf{v}-\chi \frac{\mathbf{j}}{\rho}\right) \times \mathbf{B}+\frac{\chi}{\rho} \nabla\left(P_{e}-\mu P\right)+\frac{1}{\rho} \nabla \cdot\left[\Lambda_{j v} \nabla \mathbf{v}+\Lambda_{j j} \nabla\left(\frac{\mathbf{j}}{\rho}\right)\right]
\end{aligned}
$$

where

$$
\Lambda_{j v}=-\chi\left(\Lambda_{e i}+\Lambda_{e e}\right)+\mu \chi\left(\Lambda_{i i}+\Lambda_{i e}\right)
$$

and

$$
\Lambda_{j j}=\chi^{2} \Lambda_{e e}-\mu \chi^{2}\left(\Lambda_{i i}-\Lambda_{i e}-\Lambda_{e i}\right) .
$$

To maintain a consistent ordering we let $P_{e}-\mu P \rightarrow P_{e}$ by absorbing the order $\mu$ part into a redefinition of $P_{e}$. We can also define another viscosity 2-tensor,

$$
\Pi_{j}=\Lambda_{j v} \nabla \mathbf{v}+\Lambda_{j j} \nabla\left(\frac{\mathbf{j}}{\rho}\right) .
$$

Given the above, from conservation of energy, one can get the new equation for entropy. The global entropy is defined by $\sigma=\sigma_{i}+\sigma_{e}$ just like the global internal energy density is defined as $u_{\mathrm{Vol}}=u_{i, \mathrm{Vol}}+u_{e, \mathrm{Vol}}$. This equation is a natural generalization of the entropy evolution for magnetic field-free flow (de Groot \& Mazur 1984). Because the derivation follows directly from the thermodynamic identity and the above equations, we exclude the details of the calculation, which yields the following:

$$
\frac{\partial \sigma}{\partial t}+\mathbf{v} \cdot \nabla \sigma+\nabla \cdot \mathbf{J}_{T}=\frac{1}{T} \nabla T \cdot \mathbf{J}_{T}+\frac{1}{T} \mathbf{j} \cdot \mathbf{J}_{j}+\frac{1}{T} \nabla \mathbf{v}: \Pi_{v}+\frac{1}{T} \nabla\left(\frac{\mathbf{j}}{\rho}\right): \Pi_{j},
$$

where we have defined the heat flux $\mathbf{J}_{T}=\eta_{T j} \mathbf{j}+\eta_{T T} \nabla T$ that drives the entropy. Here we have added the two mirror coefficients of conductivity. The first is the usual heat conductivity $\eta_{T T}$. This coefficient is usually defined with a factor $T$, which here for simplicity and symmetry is absorbed in the definition of the phenomenological coefficient. Indeed, it will appear that the natural variable is $\nabla(1 / T)$ and not $\nabla T$; we then add $T$ to the phenomenological coefficients to compensate. The second coefficient is the cross effect conductivity $\eta_{T j}$, which is expected to be symmetric with $\eta_{j T}$ because of the Onsager relations.

Next we specify the pressure and temperature. Just like before, we suppose there is a known total internal energy density $u_{\mathrm{Vol}}(\rho, \sigma)$, whence the temperature $T$ and total pressure $P$ are given by

$$
T=\frac{\partial u_{\mathrm{Vol}}}{\partial \sigma} \quad \text { and } \quad P=\frac{1}{\rho^{2}} \frac{\partial u_{\mathrm{Vol}}}{\partial \rho} .
$$

It remains to specify the electron pressure $P_{e}$ that appears in the Ohm's law of (2.4). In a more general study we might keep the electron entropy $\sigma_{e}$ and define it as in Kimura \& Morrison (2014). In a nondissipative study, this is not a problem since the entropies are conserved. But now they evolve and it is tricky to find out which kind of 
dissipation varies with each entropy, since some forms of dissipation are exchange terms, i.e., they exchange entropy. Indeed, the thermodynamic study has four variables that evolve with time: the two internal energies and the two entropies, which are linked by two thermodynamic identities. The usual study takes advantages of the conservation of energy to close the system. But here we still lack an energy equation. To compensate, we have chosen a common local equilibrium temperature. This is a strong hypothesis since plasmas are often not thermalized in this way. In reality, electrons would equilibrate at one rate and ions at another, with both eventually equilibrating to a common temperature. Collisional processes could be added to account for this, but if the temperatures are initially close to each other our choice should be a good approximation. Moreover, as noted above, this paper is already technical, and the techniques developed do point to the way to more complete models. Under this assumption, we suppose a known expression for the electron Helmholtz free energy density $f_{e}:=u_{e, \mathrm{Vol}}-T \sigma_{e}$, which is a function of $\left(\rho_{e}, T\right)$, and this is determined for all time by our set of equations. We then can define

$$
P_{e}=\frac{1}{\rho_{e}^{2}} \frac{\partial f_{e}}{\partial \rho_{e}} .
$$

If we no longer assume the local temperature equilibrium, the problem becomes harder. The global thermodynamic identity uses two temperatures, so the entropy evolution is more complex. We save this study for future work.

\subsection{Reduced Equations and Discussion}

As before, we eliminate $\mathbf{j}$ and $\mathbf{E}$ and write the equations in terms of $\left(\rho, \sigma, \mathbf{m}, \mathbf{B}^{*}\right)$, four evolution equations and four phenomenological equations.

The first evolution equation is mass conservation,

$$
\partial_{t} \rho+\nabla \cdot \mathbf{m}=0
$$

the second is momentum conservation,

$$
\partial_{t} \mathbf{m}+\nabla \cdot(\mathbf{m} \otimes \mathbf{v})=-\nabla P-\mu \rho \frac{\chi^{2}}{2} \nabla\left(\frac{|\mathbf{j}|^{2}}{\rho^{2}}\right)+\mathbf{j} \times \mathbf{B}^{*}+\nabla \cdot \Pi_{v} ;
$$

the third is the magnetic field evolution equation,

$$
\begin{gathered}
\partial_{t} \mathbf{B}^{*}=\nabla \times\left[\mathbf{v} \times \mathbf{B}^{*}-\frac{\chi}{\rho}(\nabla \times \mathbf{B}) \times \mathbf{B}^{*}+\mu \frac{\chi}{\rho}(\nabla \times \mathbf{B}) \times(\nabla \times \mathbf{v})\right. \\
\left.-\mathbf{J}_{j}+\frac{1}{\rho} \nabla \cdot \Pi_{j}\right]-\frac{\chi}{\rho^{2}}\left(\nabla P_{e} \times \nabla \rho\right) ;
\end{gathered}
$$

and the forth is the entropy equation,

$$
\begin{aligned}
\partial_{t} \sigma+\nabla \cdot(\sigma \mathbf{v})=\nabla \cdot \mathbf{J}_{T} & +\frac{1}{T} \nabla T \cdot \mathbf{J}_{T}+\frac{1}{T} \mathbf{j} \cdot \mathbf{J}_{j} \\
& +\frac{1}{T} \nabla \mathbf{v}: \Pi_{v}+\frac{1}{T} \nabla\left(\frac{\mathbf{j}}{\rho}\right): \Pi_{j} .
\end{aligned}
$$

On the other hand, the first two phenomenological equations determine the viscosities,

$$
\Pi_{v}=\Lambda_{v v} \nabla \mathbf{v}+\Lambda_{v j} \nabla\left(\frac{\mathbf{j}}{\rho}\right) \quad \text { and } \quad \Pi_{j}=\Lambda_{j v} \nabla \mathbf{v}+\Lambda_{j j} \nabla\left(\frac{\mathbf{j}}{\rho}\right) ;
$$

while the second two the conduction,

$$
\mathbf{J}_{j}=\eta_{j j} \mathbf{j}+\eta_{j T} \nabla T \quad \text { and } \quad \mathbf{J}_{T}=\eta_{T j} \mathbf{j}+\eta_{T T} \nabla T .
$$


Let us discuss the several dissipative effets. Some of them are usual: the tensor $\Lambda_{v v}$ is the usual viscosity that gives viscous dissipation of the velocity if this velocity has spatial variation; the matrix $\eta_{j j}$ is the usual electrical resisitivity that gives the usual Ohm's law; and the coefficient $T \eta_{T T}$ is the usual heat conductivity that gives the usual Fourier law. The other coefficients are less usual. The cross terms $\eta_{j T}$ and $\eta_{T j}$ are thermo-electric coefficients, which arise from different responses of the different particles to the gradient of the temperature. In this context, this phenomenon is called thermophoresis. More precisely, $\eta_{j T}$ gives the Soret effet, while $\eta_{T j}$ gives the Dufour effect.

The current viscosity $\Lambda_{j j}$ is predominately determined by the electron viscosity. This seems natural in the context of XMHD since the electrons do have inertia. Also, one might think there could be contributions of higher order in the exchange terms, emerging from electron-ion collisions.

According to two-fluid theory, if the electrons have viscosity, then the coefficient $\Lambda_{j j}$ will not vanish. However, in magnetofluid models this effect is neglected and does not appear to have been studied. One can understand this by roughly estimating the order of magnitude of a viscosity coefficient. Dimensionally one has $[\Lambda]=\mathrm{M} \cdot \mathrm{L}^{-1} \cdot \mathrm{T}^{-1}$. Choosing parameters as the mass density $n m_{\alpha}$, where $\alpha \in\{e, i\}$, one can estimate, with particle number density $n$, the average velocity $v_{\alpha}$ (or equivalently the thermal energy $T$ ), and the Debye length $\lambda_{D}^{2}=\frac{T}{4 \pi n e^{2}}$, which is clearly the smallest length possible here although any length will do, we obtain

$$
\left|\Lambda_{\alpha \alpha}\right| \sim m_{\alpha} n v_{\alpha} \lambda_{D} \sim n \sqrt{T m_{\alpha}} \lambda_{D}
$$

Since $n$ and $T$ are the same for both electrons and ions, one can estimate

$$
\frac{\left|\Lambda_{e e}\right|}{\left|\Lambda_{i i}\right|} \sim \sqrt{\frac{m_{e}}{m_{i}}}=\sqrt{\mu} .
$$

Physically, the idea is that because electrons are much lighter, there are more electronelectron than ion-ion collisions, mostly because they are quicker, yet these collisions contribute less to the change of momentum.

Alternatively, one can arrive at this result from the work of Braginskii (1965). Using Braginskii's coefficients $\eta_{0 e}$ and $\eta_{0 i}$ as estimates for the order of magnitude of $\Lambda_{e e}$ and $\Lambda_{i i}$, and assuming equal temperatures $T_{e}=T_{i}$, one has $\left|\Lambda_{e e}\right| /\left|\Lambda_{i i}\right| \sim \tau_{e} / \tau_{i}$, with $\tau_{\alpha}$ being the collision time for $\alpha \in\{e, i\}$; because $\tau_{\alpha} \propto \sqrt{m_{\alpha}}$ one reaches the same conclusion.

Finally, in order of magnitude, one has $\left|\Lambda_{v v}\right| \sim\left|\Lambda_{i i}\right| \sim 1$ and $\left|\Lambda_{j j}\right| \sim\left|\Lambda_{e e}\right| \sim \sqrt{\mu}$. Numerically, this is small, yet terms of this order are retained in the XMHD framework. This may explain why there appears to be no literature on this effect, even though our estimates suggest retaining this term as a higher order correction of the usual formulas. We caution that our estimates are approximate and various temperature differences for ions and electrons may also change this result. So, keeping in mind that this effect is small, we will keep it in our equations for the purposes of generality and symmetry. Getting general brackets is easier this way, since one is reminded of this symmetry, which also appears naturally in the brackets.

With regard to the cross effects, the Onsager relations and the constraint of entropy growth assure $\left|\Lambda_{i e}\right| \sim\left|\Lambda_{i e}\right| \leqslant \sqrt{\left|\Lambda_{i i}\right|\left|\Lambda_{e e}\right|}$. For usual cross effects, one may believe that these coefficients also are of order $\sqrt{\mu}$. Since these cross-effects can be interesting terms and also exhibit symmetries, we will keep them too. These cross-effects may be interesting since they provide a new way to mix the velocity field and the magnetic field, of higher derivative order and linear. Then, close to equilibrium and for fast variations, while other mixing terms may disappear, these cross effect may offer new kinds of mixing. An easy and naive example will be provided in the next subsection. 


\subsection{An Example of the Viscous Cross-Effects}

In this subsection, we will brief illustration of the effect of the viscous cross-terms by showing how they can provide an avenue for transferring mechanical energy into electromagnetic energy. The model is drastically simplified, and intended to be educational for gaining insight into the meaning of these new terms.

To this end we assume, $\Omega=\mathbb{R}_{y}^{+} \times \mathbb{R}^{2}$, with translational symmetry along the $z$-axis, i.e., we work with a 2D-fluid. All phenomenological tensors are assumed to be constant scalars and the internal energy is chosen so that $\rho$ remains constant and uniform, just like $T$. At $y=0$ we assume there is a wall that oscillates in the $x$-direction with velocity $u=u_{0} e^{i \nu t}$, with $u_{0}$ being the amplitude, assumed small, and $\nu$ is the frequency, assumed large. We work with the viscous boundary limit, close enough to the wall and with $u_{0}$ small enough to neglect all nonlinear terms. We will not exhibit the huge constraints of such hypotheses. At the beginning of this thought experiment, there is no magnetic field, and there are no outside sources, so that the electromagnetic energy is initially zero. We ask the question, upon forcing the fluid with such a sinusoidal mechanical input: What will happen?

For a classical fluid, like a plasma without the viscous cross term, the magnetic field equation states $\mathrm{d} \mathbf{B}^{*} / \mathrm{d} t=0$. Thus, the electromagnetic energy remains zero and the problem is purely mechanical. If one defines the scalar vorticity as $\omega=\hat{z} \cdot \nabla \times \mathbf{v}$, then the equation of motion becomes $\rho \partial_{t} \omega=\Lambda_{v v} \Delta \omega$. The mechanical sinosoidal input will then give rise to a sinusoidal output $\omega=\omega_{0} e^{k y+i \nu t}$, where the wavenumber $k$ will respect the dispersion relation:

$$
k=-(1 \pm i) \delta^{-1}, \quad \text { where } \quad \delta=\sqrt{\frac{2 \Lambda_{v v}}{\rho \nu}} .
$$

The quantity $\delta$ is the boundary layer thickness and $\omega_{0} \sim u_{0} / \delta$. Physically, the sinusoidal input will create oscillations in the fluid along the same direction, and this oscillation will propagate in the $y$-direction with an exponential decrease into the boundary layer.

Now, if we add the other viscous terms, which are linear, the situation changes. The linear terms are now,

$$
\begin{aligned}
\partial_{t} \omega & =\frac{\Lambda_{v v}}{\rho} \Delta \omega-\frac{\Lambda_{v j}}{\rho} \Delta^{2} B \\
\partial_{t} B^{*} & =\eta_{j j} \Delta B+\frac{\Lambda_{j v}}{\rho} \Delta \omega-\frac{\Lambda_{j j}}{\rho} \Delta^{2} B,
\end{aligned}
$$

where the components of $B^{*}$ and $B$ are along the symmetry direction. Then, the sinusoidal input appears in the magnetic field equation thanks to the $\Lambda_{j v}$ term. The solutions will then become

$$
\omega=\omega_{0} e^{k y+i \nu t} \quad \text { and } \quad B=B_{0} e^{k y+i \nu t},
$$

and the wavenumber $k$ is now given by the dispersion relation

$$
\left(\frac{\Lambda_{v v}}{\rho} k^{2}-i \nu\right)\left(\eta_{j j} k^{2}-\frac{\Lambda_{j j}}{\rho} k^{4}-i \nu\left(1-\mu \frac{\chi^{2}}{\rho} k^{2}\right)\right)+\frac{\Lambda_{v j} \Lambda_{j v}}{\rho^{2}} k^{6}=0 .
$$

Thus, one can link $\omega_{0}$ and $B_{0}$, while $\omega_{0} \sim|k| u_{0}$.

Evidently, the system of equations obtained could be studied more deeply, the above is sufficient for our purpose: How should one understand this thought experiment? The wall moves sinusoidally and the viscous boundary layer limit means the wall will drive the fluid with it. Then, these oscillations propagate in the $y$-direction, so the wall moves a column 
of fluid. And it does so with some effectiveness, parametrized by the viscous coefficient. But which fluid is drifted? The ions or the electrons? In fact, both, but not with the same effectiveness, i.e., not with the same force. Thus, electrons and ions oscillate, but not at the same amplitude, thereby creating a current. If one looks at the scene from the ions' point of view, one would see electrons oscillating. Indeed, this interface effect is creating an alternating electric current from a mechanical input. Thus, we have a sort of wall-driven dynamo effect.

\section{Thermodynamic Theory of Dissipative Brackets}

\subsection{From Nonequilibrium Thermodynamics to Dissipative Brackets}

While thermodynamics historically deals with equilibrium states, nonequilibrium thermodynamics is concerned with systems close to thermal equilibrium and implements irreversible processes (de Groot \& Mazur 1984; Gav-Balmaz \& Yoshimura 2017a.b). In developing such a theory, the first step is to write a thermodynamic identity

$$
\mathrm{d} \sigma=\sum_{\alpha} X^{\alpha} \mathrm{d} \zeta_{\alpha}
$$

where, as before, $\sigma$ is the entropy density and the $\zeta_{\alpha}$ are densities associated with conserved extensive properties, with $X^{\alpha}=\partial \sigma / \partial \zeta_{\alpha}$. The $\zeta_{\alpha}$ will eventually be used to define a convenient set of dynamical variables. (See section 3.2.) One then has conservation equations for all the densities,

$$
\partial_{t} \zeta_{\alpha}+\nabla \cdot \mathbf{J}_{\alpha}=0
$$

where $\mathbf{J}_{\alpha}$ is an unknown flux associated with $\zeta_{\alpha}$. Given the above, the evolution of the entropy is determined by the equation of motion

$$
\partial_{t} \sigma+\nabla \cdot \mathbf{J}_{T}=\sum_{\alpha} \mathbf{J}_{\alpha} \cdot \nabla X^{\alpha} ; \quad \mathbf{J}_{T}=\sum_{\alpha} X^{\alpha} \mathbf{J}_{\alpha}
$$

and $\nabla X^{\alpha}$ is called the affinity associated with the density and flux labeled by $\alpha$.

It remains to determine the fluxes $\mathbf{J}_{\alpha}$. Close to equilibrium, one typically assumes linear response:

$$
\mathbf{J}_{\alpha}=\sum_{\beta} L_{\alpha \beta} \nabla X^{\beta}
$$

for any $\alpha$. Up to this point, $L$ could be any tensor. But of course, physics constrains its form. Because of Onsager's relations, $L$ should be symmetric. Plus, the growth of entropy is assured if and only if $L$ has nonnegative eigenvalues. We make an important connection by associating dynamics generated with a bracket with the tensor $L$. That is, we first prove a formal equivalence between the classical out-of-equilibrium thermodynamics and a subclass of metriplectic dynamical systems. Thus, we show that the pseudometric nature of the dissipative bracket, usually an ad hoc hypothesis, is the exact transcription of the well-known second law of thermodynamics and Onsager's relations through this equivalence.

It is then natural to define the phase space, a vector space of functions on $\Omega$, that has the basis $\left\{\zeta_{\alpha}, \forall \alpha\right\}$, in which the entropy is geometrically constructed; thus, the form of (3.1) would be maintained for another choice of basis besides $\left\{\zeta_{\alpha}, \forall \alpha\right\}$ provided it defines a suitable set of thermodynamic variables (Callen 1960). Then, one can decompose the phase space into a part defined by the kernel of $L$ and a subspace where $L$ defines a metric. 
To see how $L$ is related to a bracket on the phase space, let us rewrite the evolution equations, at a space point $\mathbf{x}$ and time $t$, as follows:

$$
\begin{aligned}
\partial_{t} \zeta_{\alpha}(\mathbf{x}, t) & =-\nabla \cdot \mathbf{J}_{\alpha}(\mathbf{x}, t)=-\nabla \cdot\left[L_{\alpha \beta}(\mathbf{x}, t) \nabla\left(\frac{\partial \sigma}{\partial \zeta_{\beta}}\right)(\mathbf{x}, t)\right] \\
& =-\int_{\Omega} \mathrm{d}^{3} y \delta_{\Omega}(\mathbf{x}-\mathbf{y}) \nabla \cdot\left[L_{\alpha \beta}(\mathbf{y}, t) \nabla\left(\frac{\partial \sigma}{\partial \zeta_{\beta}}\right)(\mathbf{y}, t)\right] \\
& =\int_{\Omega} \mathrm{d}^{3} y\left[\nabla\left(\delta_{\Omega}(\mathbf{x}-\mathbf{y})\right) L_{\alpha \beta}(\mathbf{y}, t) \nabla\left(\frac{\partial \sigma}{\partial \zeta_{\beta}}\right)(\mathbf{y}, t)\right] \\
& =\int_{\Omega} \mathrm{d}^{3} y\left[\nabla\left(\frac{\delta \zeta_{\alpha}(\mathbf{x}, t)}{\delta \zeta_{\gamma}(\mathbf{y}, t)}\right) L_{\gamma \beta}(\mathbf{y}, t) \nabla\left(\frac{\delta \mathcal{S}(t)}{\delta \zeta_{\beta}(\mathbf{y}, t)}\right)\right],
\end{aligned}
$$

where have been used repeated index notation for summation over $\beta$ and $\gamma$ and $\delta_{\Omega}(\mathbf{x}-\mathbf{y})$ is the Dirac delta function. Proceeding from (3.2) one easily recognizes a bracket, because the $\left\{\zeta_{\alpha}, \forall \alpha\right\}$ constitutes a basis of the phase space. To make this clearer, we write the entropy evolution equation as follows:

$$
\begin{aligned}
\partial_{t} \sigma(\mathbf{x}, t)= & -\nabla \cdot \mathbf{J}_{T}(\mathbf{x}, t)+\mathbf{J}_{\alpha} \cdot \nabla\left(\frac{\partial \sigma}{\partial \zeta_{\alpha}}\right)(\mathbf{x}, t) \\
= & -\nabla \cdot\left[\frac{\partial \sigma}{\partial \zeta_{\alpha}}(\mathbf{x}, t) L_{\alpha \beta}(\mathbf{x}, t) \nabla\left(\frac{\partial \sigma}{\partial \zeta_{\beta}}\right)(\mathbf{x}, t)\right] \\
& +\nabla\left(\frac{\partial \sigma}{\partial \zeta_{\alpha}}\right)(\mathbf{x}, t) L_{\alpha \beta}(\mathbf{x}, t) \nabla\left(\frac{\partial \sigma}{\partial \zeta_{\beta}}\right)(\mathbf{x}, t) \\
= & -\frac{\partial \sigma}{\partial \zeta_{\alpha}}(\mathbf{x}, t) \nabla \cdot\left[L_{\alpha \beta}(\mathbf{x}, t) \nabla\left(\frac{\partial \sigma}{\partial \zeta_{\beta}}\right)(\mathbf{x}, t)\right] \\
= & \int_{\Omega} \mathrm{d}^{3} y\left[\nabla\left(\frac{\partial \sigma}{\partial \zeta_{\alpha}}(\mathbf{y}, t) \delta_{\Omega}(\mathbf{x}-\mathbf{y})\right) L_{\alpha \beta}(\mathbf{y}, t) \nabla\left(\frac{\partial \sigma}{\partial \zeta_{\beta}}(\mathbf{y}, t)\right)\right] \\
= & \int_{\Omega} \mathrm{d}^{3} y\left[\nabla\left(\frac{\delta \sigma(\mathbf{x}, t)}{\delta \zeta_{\alpha}(\mathbf{y}, t)}\right) L_{\alpha \beta}(\mathbf{y}, t) \nabla\left(\frac{\delta \mathcal{S}(t)}{\delta \zeta_{\beta}(\mathbf{y}, t)}\right)\right]
\end{aligned}
$$

Thus, the dynamics of out-of-equilibrium thermodynamics on the phase space can be express with a symmetric bracket. Namely, for any two functionals $f, g$, we define the bracket

$$
(f, g):=\frac{1}{\mathcal{T}} \int_{\Omega} \mathrm{d}^{3} y \nabla\left(\frac{\delta f}{\delta \zeta_{\alpha}(\mathbf{y})}\right) L_{\alpha \beta} \nabla\left(\frac{\delta g}{\delta \zeta_{\beta}(\mathbf{y})}\right)
$$

Here the phenomenological tensor $L_{\alpha \beta}$ is written with explicit subscripts $\alpha$ and $\beta$ denoting the processes, while other tensorial indices are suppressed. Let us remark that it is independent of the basis $\left\{\zeta_{\alpha}, \forall \alpha\right\}$. Indeed, the functional derivatives can be seen as functional gradients, and both functional gradients are contracted thanks to the pseudometric $L$. We then deal with purely geometrical objects. Similarly, if $\zeta_{\alpha}$ is an $a$-tensor and $\zeta_{\beta}$ is an $b$-tensor, then $L_{\alpha \beta}$ is an $(a+b+2)$-tensor. Plus, notice that thanks to the Onsager relations, the bracket is symmetric. Finally, one can write the evolution of any functional $f$ as

$$
\frac{\mathrm{d} f}{\mathrm{~d} t}=\mathcal{T}(f, \mathcal{S})
$$

Our construction above shows that the dissipative brackets are completely natural for nonequilibrium thermodynamics, just like Poisson brackets are natural for Hamiltonian dynamics. Above, we have explicitly derived a general dissipative bracket, apparently for 
the first time, from basic thermodynamic first principles. This bracket is general and covers existing fluid-like theories of nonequilibrium thermodynamics such as that originally given by Morrison (1984b) and then others (Materassi \& Tassi 2012; Grmela \& Öttinger $1997 a$; Edwards 1998).

Consider now the role of entropy $\mathcal{S}$. It plays a role counterpart to the role of the Hamiltonian in analytical mechanics; however, of course here, it is not a conserved quantity. On the contrary, the nonnegativity of the pseudometric $L$ assures the entropy growth, i.e. the second law of thermodynamics. We have seen in noncanonical Hamiltonian mechanics that using $\sigma$ as a variable was useful because its integral $\mathcal{S}$, being a Casimir invariant, is conserved. Another variable that is very important, but not often a basic dynamical variable, is $\varepsilon$, the energy density, since it appears in the Hamiltonian that generates the dynamics. In thermodynamics, $\sigma$ is no longer a natural independent variable. But $\varepsilon$, since the energy is preserved, is a natural variable for the thermodynamic identity and then for the basis. While $\sigma$ now appears through the entropy $\mathcal{S}$ that generates the dynamics. In brief, the roles of $\varepsilon$ and $\sigma$ are interchanged. Thus, there are natural variables for the basis of the phase space, but these are different in the Hamiltonian and thermodynamic points of view. Nevertheless, one can change variables, thanks to the thermodynamic identity, and obtain a bracket in any complete set of phase space variables.

Finally we ask: What about the first law of thermodynamics? Using $\varepsilon$ as a basic variable in the thermodynamic framework makes it clear. Indeed, since $\delta \mathcal{H} / \delta \varepsilon$ is unity, a uniform constant, and the other elements of the basis are independent of $\varepsilon$, one gets for any functional $f$,

$$
(f, \mathcal{H})=0 .
$$

This is not a coincidence, since by construction the nonequilibrium thermodynamics conserves $\int_{\Omega} \zeta_{\alpha}$ for any $\alpha$, and $\varepsilon$ is chosen as one of the $\zeta_{\alpha}$ 's. Therefore, by construction, our dissipative bracket has a strong formulation of the first law of thermodynamics. Indeed it has all of the properties given in Morrison (1984a,, , 1986), including bilinearity, symmetry, and degeneracy. Coupling such a bracket with the associated noncanonical Poisson bracket gives a metriplectic dynamical system.

\subsection{Application to Hydrodynamics}

For hydrodynamics our formalism reduces to a single fluid, without the electromagnetic effects, and the equations of this model are

$$
\begin{aligned}
\frac{\mathrm{d} \rho}{\mathrm{d} t} & =-\rho \nabla \cdot \mathbf{v} \\
\rho \frac{\mathrm{d} \mathbf{v}}{\mathrm{d} t} & =-\nabla P+\nabla \cdot(\Lambda \nabla \mathbf{v}) \\
\frac{\mathrm{d} \sigma}{\mathrm{d} t} & =\nabla \cdot\left(\frac{1}{T} \kappa \nabla T\right)+\frac{1}{T^{2}} \nabla T \cdot \kappa \nabla T+\frac{1}{T} \nabla \mathbf{v}: \Lambda \nabla \mathbf{v},
\end{aligned}
$$

where $\Lambda$ is the viscosity, a 4 -tensor, and $\kappa$ the heat conductivity, in general a 2 -tensor.

We now apply our new formulation to the fluid, whose variables are $(\varepsilon, \rho, \mathbf{m})$. Indeed, these variables are independent, they specify the state of the fluid, and they are conserved densities; respectively, the total energy $\mathcal{H}$, the global momentum $\mathcal{P}$, and the total mass $\mathcal{M}$ are constants of motion:

$$
\mathcal{H}=\int_{\Omega} \varepsilon, \quad \mathcal{P}=\int_{\Omega} \mathbf{m}, \quad \text { and } \quad \mathcal{M}=\int_{\Omega} \rho .
$$

Our construction guarantees that these quantities will remain constant. If $u$ is the specific 
internal energy, the local energy density is

$$
\varepsilon=\frac{|\mathbf{m}|^{2}}{2 \rho}+\rho u(\rho, s),
$$

where $s$ is the specific entropy and $\sigma=\rho s$. The thermodynamic identity reads $\mathrm{d} u=$ $T \mathrm{~d} s+P \mathrm{~d} \rho / \rho^{2}$, which upon changing variables gives

$$
T \mathrm{~d} \sigma=\mathrm{d} \varepsilon-\mathbf{v} \cdot \mathrm{d} \mathbf{m}-g \mathrm{~d} \rho,
$$

where $g$ is a modified specific Gibbs free energy, namely $g:=u-T s+P / \rho-|\mathbf{v}|^{2} / 2$. Its differential is then $\mathrm{d} g=-s \mathrm{~d} T+\mathrm{d} P / \rho-\mathbf{v} \cdot \mathrm{d} \mathbf{v}$, so that $g(T, P, \mathbf{v})$ is an extensive quantity with intensive arguments and, consequently, vanishes. Let us remark that in this paper we do not consider chemical reactions or particle creation/annihilation - for such cases this free energy would not vanish. Finally, the phase space for thermodynamics is smaller than that for the Hamiltonian case because $\rho$ does not appear in the thermodynamic identity and may be ignored. The thermodynamic variables then are $(\varepsilon, \mathbf{m})$ and the thermodynamic identity is

$$
T \mathrm{~d} \sigma=\mathrm{d} \varepsilon-\mathbf{v} \cdot \mathrm{d} \mathbf{m} .
$$

From this thermodynamic identity, one can see that there will be two irreversible responses, linked to $\varepsilon$ and $\mathbf{m}$, that could be expressed with the affinities $\nabla T$ and $\nabla \mathbf{v}$. These dissipation processes are, respectively, the heat conduction and the viscosity. We are set to proceed, but there are two complications. First, the natural affinity associated with $\varepsilon$ is $\nabla(1 / T)$, but this choice implies some factors of $T$ will appear. Second, the natural affinity of $\mathbf{m}$ is $-\nabla(\mathbf{v} / T)$, but this may create cross effects. Since we know because of space-parity symmetry no such cross effects exist between the affinities $\nabla T$ and $\nabla \mathbf{v}$, we must destroy them using nondiagonal terms of the tensor $L$. This is a strange constraint that is certainly linked with the choice of the basis.

To get the expression for $L$, one can look at the various fluxes and compare them with the usual notations (de Groot \& Mazur 1984). For $\mathbf{m}$, the flux is the opposite of the usual viscous tensor, viz.,

$$
\Pi_{v}=L_{m m} \nabla\left(\frac{\mathbf{v}}{T}\right)-L_{m \varepsilon} \nabla\left(\frac{1}{T}\right)=\Lambda \nabla \mathbf{v},
$$

whence $L_{m m}=T \Lambda$ and $L_{m \varepsilon}=T \Lambda \mathbf{v}$. On the other hand, the energy flux is

$$
\mathbf{J}_{\varepsilon}=-L_{\varepsilon m} \nabla\left(\frac{\mathbf{v}}{T}\right)+L_{\varepsilon \varepsilon} \nabla\left(\frac{1}{T}\right)=\mathbf{v} \cdot \Pi_{v}+\kappa \nabla T,
$$

whence $L_{\varepsilon m}=T \mathbf{v} \cdot \Lambda$ and $L_{\varepsilon \varepsilon}=T^{2} \kappa+\mathbf{v} \cdot \Lambda \mathbf{v}$. Thus, $L$ is effectively symmetric and it is easy to check that we have the correct heat flux,

$$
\mathbf{J}_{T}=\frac{1}{T} \mathbf{J}_{\varepsilon}+\mathbf{v} \cdot \Pi_{v}=\frac{1}{T} \kappa \nabla T .
$$

Having proceeded in this systematic way, the bracket on any functionals $f$ and $g$ is immediate:

$$
\begin{gathered}
(f, g)=\frac{1}{\mathcal{T}} \int_{\Omega} \mathrm{d}^{3} y\left(\nabla\left(\frac{\delta f}{\delta \varepsilon(\mathbf{y})}\right) \cdot\left[\left(T^{2} \kappa+\mathbf{v} \cdot \Lambda \mathbf{v}\right) \nabla\left(\frac{\delta g}{\delta \varepsilon(\mathbf{y})}\right)+T \mathbf{v} \cdot \Lambda \nabla\left(\frac{\delta g}{\delta \mathbf{m}(\mathbf{y})}\right)\right]\right. \\
\left.+\nabla\left(\frac{\delta f}{\delta \mathbf{m}(\mathbf{y})}\right):\left[T \Lambda \mathbf{v} \otimes \nabla\left(\frac{\delta g}{\delta \varepsilon(\mathbf{y})}\right)+T \Lambda \nabla\left(\frac{\delta g}{\delta \mathbf{m}(\mathbf{y})}\right)\right]\right)
\end{gathered}
$$


We know this bracket is a metriplectic bracket that preserves the desired quantity, it is symmetric, and it is positive.

What about the known dissipative bracket of hydrodynamics given by Morrison $(1984 b)$ ? To compare we transform back to the more usual fluid dynamical variables of fluid mechanics, $(\varepsilon, \mathbf{m}, \rho) \longrightarrow(\sigma, \mathbf{m}, \rho)$. Via the chain rule the functional derivatives satisfy

$$
\frac{\delta f}{\delta \varepsilon} \longrightarrow \frac{\partial \sigma}{\partial \varepsilon} \frac{\delta f}{\delta \sigma}=\frac{1}{T} \frac{\delta f}{\delta \sigma} \quad \text { and } \quad \frac{\delta f}{\delta \mathbf{m}} \longrightarrow \frac{\delta f}{\delta \mathbf{m}}+\frac{\partial \sigma}{\partial \mathbf{m}} \frac{\delta f}{\delta \sigma}=\frac{\delta f}{\delta \mathbf{m}}-\frac{\mathbf{v}}{T} \frac{\delta f}{\delta \sigma}
$$

Using

$$
\nabla\left(\frac{\delta f}{\delta \mathbf{m}}-\frac{\mathbf{v}}{T} \frac{\delta f}{\delta \sigma}\right)=\nabla\left(\frac{\delta f}{\delta \mathbf{m}}\right)-\frac{1}{T} \frac{\delta f}{\delta \sigma} \nabla \mathbf{v}-\nabla\left(\frac{1}{T} \frac{\delta f}{\delta \sigma}\right) \otimes \mathbf{v},
$$

it is seen that the last term compensates the cross terms, simplifying the heat part of the bracket, yielding,

$$
\begin{aligned}
(f, g)= & \int_{\Omega} \mathrm{d}^{3} y \frac{T}{\mathcal{T}}\left[T \nabla\left(\frac{1}{T} \frac{\delta f}{\delta \sigma(\mathbf{y})}\right) \cdot \kappa \nabla\left(\frac{1}{T} \frac{\delta g}{\delta \sigma(\mathbf{y})}\right)\right. \\
& \left.+\left(\nabla\left(\frac{\delta f}{\delta \mathbf{m}(\mathbf{y})}\right)-\frac{1}{T} \frac{\delta f}{\delta \sigma(\mathbf{y})} \nabla \mathbf{v}\right): \Lambda\left(\nabla\left(\frac{\delta g}{\delta \mathbf{m}(\mathbf{y})}\right)-\frac{1}{T} \frac{\delta g}{\delta \sigma(\mathbf{y})} \nabla \mathbf{v}\right)\right]
\end{aligned}
$$

the bracket given by Morrison $(1984 b)$.

To summarize, in this section we have developed a systematic way to construct dissipative brackets, and we showed that this method reproduces the known bracket for hydrodynamics. In the next section we will use the method to derive a bracket for XMHD. There we will change some notation, i.e., we write for the 2-tensor $\eta_{T T}:=\kappa / T$ and the 4 -tensor $\Lambda_{v v}:=\Lambda$.

\section{Derivation of the Brackets of Dissipative Extended Magnetohydrodynamics}

\subsection{Thermodynamics}

We now return to XMHD. Recall, for this theory the energy density $\varepsilon$ is given by the expression of (1.3) with $\mathbf{B}^{*}$ given by (1.1). For dissipative XMHD, this quantity needs to be incorporated into the theory via an appropriate choice of a magnetic conserved quantity. Then, we can choose the associated magnetic variable and use directly the general bracket theory derived in section 3 .

Since $\varepsilon$ now depends on the magnetic field, the thermodynamic identity will be modified accordingly. First, note that the global momentum $\mathcal{P}=\int_{\Omega} \mathbf{m}$ is conserved, consistent with the Galilean symmetry as realized by the noncanonical bracket (Morrison 1982). Because of quasineutrality the local momentum $\mathbf{m}$ has no magnetic (vector potential) piece (as explained in Keramidas Charidakos et al. (2014)), and so remains equal to $\rho \mathbf{v}$. Consequently, $\mathcal{P}$ will not participate in the magnetic part of the dissipation. One can check easily from the equations of dissipative XMHD of section 2 that the integrated drifted magnetic field $\int_{\Omega} \mathbf{B}^{*}$ is preserved. Thus, we will use the coordinates $\left(\varepsilon, \rho, \mathbf{m}, \mathbf{B}^{*}\right)$ and express the thermodynamic identity in terms of these variables.

One may ask why $\int_{\Omega} \mathbf{B}^{*}$ should be conserved, which unlike other conserved quantities does not come from deeper insight such as Galilean invariance. To explain this, let us first digress for a moment and address why $\int_{0} \mathbf{B}$ is conserved for ordinary magnetohydrodynamics, a fact pointed out as early as Morrison (1982) that did not seem to be well 
known. To interpret this conservation law, consider the postulate of conservation of the magnetic flux of any surface moving with the velocity field $\mathbf{v}$. First, remember that the magnetic field is a pseudovector (vector density), which is more naturally re-expressed as a 2 -form. In local coordinates, this 2 -form, denoted $\mathfrak{B}$, can be written as $\mathfrak{B}_{a b}=\varepsilon_{a b c} \mathbf{B}_{c}$, where $\varepsilon_{a b c}$ is the Levi-Civita tensor. Thus, the magnetic flux through a surface $\Sigma$ with unit normal $\mathbf{n}$ is $\int_{\Sigma} \mathbf{B} \cdot \mathbf{n}=\int_{\Sigma} i^{*} \mathfrak{B}$, where $i^{*}$ is the pull back of the inclusion $\Sigma \subset \Omega$ that chooses the associated coordinate of the 2 -form $\mathfrak{B}$. Given the assumption that the flux through a surface is preserved when advected by $\mathbf{v}$, not forgetting that the surface $\Sigma$ moves following this vector field, we get the equation

$$
\frac{\mathrm{d}}{\mathrm{d} t} \int_{\Sigma} i^{*} \mathfrak{B}=\int_{\Sigma} i^{*}\left(\partial_{t} \mathfrak{B}+£_{\mathbf{v}} \mathfrak{B}\right)=0,
$$

where $£_{\mathbf{v}}$ is the Lie derivative generated by the vector field $\mathbf{v}$. So, our postulate is equivalent to the local Lie dragging equation

$$
\partial_{t} \mathfrak{B}+£_{\mathbf{v}} \mathfrak{B}=0 .
$$

Now, choose some fixed direction to evaluate the magnetic field, that is, a 1 -form $\theta$. We can restrict ourselves to closed 1 -forms, i.e., $\mathrm{d} \theta=0$. Remembering that the magnetic field being divergence-free means $\mathrm{d} \mathfrak{B}=0$, and using the Cartan formula and Stokes theorem, we have the identity

$$
\int_{\Omega} \mathfrak{B} \wedge £_{\mathbf{v}} \theta=\int_{\Omega} \mathfrak{B} \wedge \mathrm{d} i_{\mathbf{v}} \theta=\int_{\Omega} \mathrm{d}\left(\mathfrak{B} \wedge i_{\mathbf{v}} \theta\right)-\int_{\Omega}(\mathrm{d} \mathfrak{B}) \wedge i_{\mathbf{v}} \theta=0,
$$

where $i_{\mathbf{v}}$ is the interior product by $\mathbf{v}$. The integral of the $\theta$-component of the magnetic field is $\int_{\Omega} \theta(\mathbf{B})=\int_{\Omega} \mathfrak{B} \wedge \theta$. One can interpret this as a global flux, the sum of the fluxes through the local surfaces normal to $\theta$. Using that the vector field $\mathbf{v}$ preserves $\Omega$, then that $\theta$ is fixed and satisfies equation (4.2), the evolution of this property is

$$
\begin{aligned}
\frac{\mathrm{d}}{\mathrm{d} t} \int_{\Omega} \theta(\mathbf{B}) & =\int_{\Omega}\left(\partial_{t}(\mathfrak{B} \wedge \theta)+£_{\mathbf{v}}(\mathfrak{B} \wedge \theta)\right) \\
& =\int_{\Omega}\left(\partial_{t} \mathfrak{B}+£_{\mathbf{v}} \mathfrak{B}\right) \wedge \theta=0 .
\end{aligned}
$$

Finally, saying that this is true for any $\theta$ is just saying that $\int_{\Omega} \mathbf{B}$ is preserved, which is exactly what we wanted. Note, here the vector field $\mathbf{v}$ could have been any vector field, and need not be the physical velocity field, since we only used that it preserves the domain $\Omega$.

The flux conservation assumption is central for magnetohydrodynamics with the velocity field being the Lie dragging field v, which is Alfven's well-known frozen-in property (e.g., Kampen \& Felderhof (1967)). Nevertheless, this is no longer true for $\mathrm{XMHD}$, since neither $\mathbf{B}$ nor $\mathbf{B}^{*}$ are advected by $\mathbf{v}$. Yet, the conservation of $\int_{\Omega} \mathbf{B}^{*}$ can be seen by using modified velocity fields. Indeed, according to D'Avignon et al. (2016) (and with their notation), the drifted magnetic field can be decomposed into the following form:

$$
\mathbf{B}^{*}=\frac{\beta_{-} \mathbf{B}_{+}-\beta_{+} \mathbf{B}_{-}}{\beta_{-}-\beta_{+}},
$$

where $\beta_{ \pm}$are scalar constants and $\mathbf{B}_{ \pm}$are modified 'magnetic fields' that satisfy equation (4.1) with modified velocities $\mathbf{v}_{ \pm}$. Thus, the integrals of $\mathbf{B}_{ \pm}$are preserved, and by linearity, the integral $\int_{\Omega} \mathbf{B}^{*}$ is too.

Given that we have settled on our magnetic conserved quantity, let us proceed with obtaining the dissipative bracket. To this end we will need that the variation of $\mathbf{B}^{*}$ can 
be expressed as

$$
\delta \mathbf{B}^{*}=\delta \mathbf{B}+\mu \chi^{2} \nabla \times\left(\frac{1}{\rho} \nabla \times \delta \mathbf{B}\right)-\mu \chi^{2} \nabla \times\left(\frac{\mathbf{j}}{\rho^{2}} \delta \rho\right) .
$$

Remembering that variations will be integrated, we can directly write, using integration by part, the following:

$$
\begin{aligned}
\mathbf{B} \cdot \delta \mathbf{B}^{*} & =\mathbf{B} \cdot \delta \mathbf{B}+\mu \chi^{2} \mathbf{B} \cdot \nabla \times\left(\frac{1}{\rho} \nabla \times \delta \mathbf{B}\right)-\mu \chi^{2} \mathbf{B} \cdot \nabla \times\left(\frac{\mathbf{j}}{\rho^{2}} \delta \rho\right) \\
& =\mathbf{B}^{*} \cdot \delta \mathbf{B}-\mu \chi^{2}\left(\frac{|\mathbf{j}|}{\rho}\right)^{2} \delta \rho
\end{aligned}
$$

where the second equality is modulo a total divergence, and similarly, since here differentials and variations are the same,

$$
\mathrm{d}\left(\frac{\mathbf{B} \cdot \mathbf{B}^{*}}{2}\right)=\mathbf{B} \cdot \mathrm{d} \mathbf{B}^{*}+\mu \chi^{2}\left(\frac{|\mathbf{j}|}{\rho}\right)^{2} \mathrm{~d} \rho .
$$

Using (4.4) and (1.3) we now can write the following thermodynamic identity:

$$
T \mathrm{~d} \sigma=\mathrm{d} \varepsilon-\mathbf{v} \cdot \mathrm{d} \mathbf{m}-\mathbf{B} \cdot \mathrm{d} \mathbf{B}^{*}-g^{*} \mathrm{~d} \rho,
$$

where $g^{*}:=u-T s+P / \rho-|\mathbf{v}|^{2} / 2+\mu \chi^{2}(|\mathbf{j}| / \rho)^{2}$ is a modified specific Gibbs free energy. Thus, its natural variables are $g^{*}(T, P, \mathbf{v}, \mathbf{j} / \rho)$ or equivalently $g^{*}\left(T, P, \mathbf{v}_{i}, \mathbf{v}_{e}\right)$. An extensivity/intensity argument like that of section 3.2 shows again $g^{*}=0$. Thus, the thermodynamic identity is given by

$$
T \mathrm{~d} \sigma=\mathrm{d} \varepsilon-\mathbf{v} \cdot \mathrm{d} \mathbf{m}-\mathbf{B} \cdot \mathrm{d} \mathbf{B}^{*} .
$$

Neglecting the factors of $T$ discussed in section 3.2, which we will return to, we would conclude that the response to $\mathbf{m}$ is $\nabla \mathbf{v}$, the gradient of the thermodynamic dual and, similarly, the response to $\mathbf{B}^{*}$ would be $\nabla \mathbf{B}$. One might think that this latter response is a 2-tensor; actually, it is not because $\mathbf{B}$, as noted above, is not a vector but a pseudovector or more naturally a 2 -form, with the constraint $\nabla \cdot \mathbf{B}=0$. Then, it is natural to think that this constraint will reduce the size of the response. Indeed, we will see that the response will be $\nabla \times \mathbf{B}=\mathbf{j}$, which is a 1-tensor, and a special case of the global 2-tensor, if we re-write the phenomenological tensor.

There is yet another complication. From the thermodynamic identity (4.5), we easily see that the response to $\varepsilon$ will create heat conduction, that the response to $\mathbf{B}^{*}$ will create electrical resistivity, and cross terms can, of course, easily be added. Also we see that the response to $\mathbf{m}$ will create velocity viscosity, but what about current viscosity? In fact, in the energy expression there is a term with $\mathbf{B}$, the magnetic energy, and so conduction, and a term in $\mathbf{j}$, the inertia of electrons, and so the current viscosity. So, what happened? We reduced variables to eliminate $\mathbf{j}$, thanks to Ampere's law. In this way we hid this effect. How should we manage this situation? We will add a new affinity, one that will create this current viscosity. The original energy had a term with $|\mathbf{j}|^{2} / \rho$, which would create an affinity $\nabla(\mathbf{j} / \rho)$, which is exactly what appears in the equations. Thus, we are finally let to the following list of affinities:

$$
\nabla\left(\frac{\delta \mathcal{S}}{\delta \varepsilon}\right) ; \quad \nabla\left(\frac{\delta \mathcal{S}}{\delta \mathbf{m}}\right) ; \quad \nabla \times\left(\frac{\delta \mathcal{S}}{\delta \mathbf{B}^{*}}\right) ; \quad \nabla\left(\frac{1}{\rho} \nabla \times\left(\frac{\delta \mathcal{S}}{\delta \mathbf{B}^{*}}\right)\right) .
$$

Now, let us return to the problem involving $\nabla T$ encountered in the hydrodynamic case. Recall, the natural variables led to the bad effect of generating a cross term with 
$\nabla T$ that must be compensated through cross terms. But our new affinity is of second order and so will create cross terms of second order in $T$. To compensate for these bad terms, we have to have a new affinity of second order in $T$. Upon examination of these cross terms, we see that the needed affinity will be

$$
\nabla\left(\frac{1}{\rho} \mathbf{B} \times \nabla\left(\frac{\delta \mathcal{S}}{\delta \varepsilon}\right)\right)
$$

One could consider a general second derivative of the temperature and modify the phenomenological tensor as a consequence, but for simplicity we will directly use the easiest affinity.

\subsection{Determination of the Phenomenological Tensor}

At this point, there are two paths to get the dissipative brackets of the theory. First, in complete analogy with the hydrodynamic case studied in section 3. we can identify the various coefficients of the phenomenological tensor from the known flux expressions derived in section 2. Then, we have an expression for the dissipative brackets that can easily be changed back to the usual set of variables. Alternatively, we can first change variables of the affinity expressions and then identify the phenomenological tensors through the known equations of the model. The advantage of this second method is that in the dynamical variables, there are no tricky cross-effects due to the gradient of temperature. For this reason we will follow the second method, buttressed by our experience with the hydrodynamic example, and find it to be an easier calculation. Both calculations lead to the same conclusion.

With the change of variables $\left(\varepsilon, \rho, \mathbf{m}, \mathbf{B}^{*}\right) \longrightarrow\left(\sigma, \rho, \mathbf{m}, \mathbf{B}^{*}\right)$, the functional derivatives of any functional $f$ become

$$
\frac{\delta f}{\delta \varepsilon} \longrightarrow \frac{1}{T} \frac{\delta f}{\delta \sigma} ; \quad \frac{\delta f}{\delta \mathbf{m}} \longrightarrow \frac{\delta f}{\delta \mathbf{m}}-\frac{\mathbf{v}}{T} \frac{\delta f}{\delta \sigma} ; \quad \frac{\delta f}{\delta \mathbf{B}^{*}} \longrightarrow \frac{\delta f}{\delta \mathbf{B}^{*}}-\frac{\mathbf{B}}{T} \frac{\delta f}{\delta \sigma} .
$$

The interesting affinities in terms of the new variables will change and, just like for the hydrodynamic case, we develop them with the temperature away from the derivatives. Developed in this way, we directly see the cross effect that we want to vanish.

Now we list the terms for any functional $f$. First, we have the $\varepsilon$ and $\mathbf{m}$ responses, which are the same as those for hydrodynamics,

$$
\nabla\left(\frac{\delta f}{\delta \varepsilon}\right) \longrightarrow \nabla\left(\frac{1}{T} \frac{\delta f}{\delta \sigma(\mathbf{y})}\right)
$$

and

$$
\nabla\left(\frac{\delta f}{\delta \mathbf{m}}\right) \longrightarrow \nabla\left(\frac{\delta f}{\delta \mathbf{m}}\right)-\frac{1}{T} \frac{\delta f}{\delta \sigma} \nabla \mathbf{v}-\nabla\left(\frac{1}{T} \frac{\delta f}{\delta \sigma}\right) \otimes \mathbf{v} .
$$

Similarly, the resistivity will arise from the magnetic response,

$$
\begin{aligned}
\nabla \times\left(\frac{\delta f}{\delta \mathbf{B}^{*}}\right) \longrightarrow & \nabla \times\left(\frac{\delta f}{\delta \mathbf{B}^{*}}\right)-\frac{1}{T} \frac{\delta f}{\delta \sigma} \nabla \times \mathbf{B}-\nabla\left(\frac{1}{T} \frac{\delta f}{\delta \sigma}\right) \times \mathbf{B} \\
& =\nabla \times\left(\frac{\delta f}{\delta \mathbf{B}^{*}}\right)-\frac{1}{T} \frac{\delta f}{\delta \sigma} \mathbf{j}+\mathbf{B} \times \nabla\left(\frac{1}{T} \frac{\delta f}{\delta \sigma}\right) .
\end{aligned}
$$




\begin{tabular}{|c|c|c|c|}
\hline Response to $f$ & \multirow{2}{*}{$\frac{\text { Explicit formula }}{\nabla\left(\frac{1}{T} \frac{\delta f}{\delta \sigma}\right)}$} & \multicolumn{2}{|c|}{$\mid$ Geometrical type $\mid$ Index for $\widehat{L} \mid$} \\
\hline$R_{T}(f)$ & & 1-tensor & $T$ \\
\hline$R_{v}(f)$ & $\nabla\left(\frac{\delta f}{\delta \mathbf{m}}\right)-\frac{1}{T} \frac{\delta f}{\delta \sigma} \nabla \mathbf{v}$ & 2 -tensor & $v$ \\
\hline$R_{B}(f)$ & $\nabla \times\left(\frac{\delta f}{\delta \mathrm{B}^{*}}\right)-\frac{1}{T} \frac{\delta f}{\delta \sigma} \mathbf{j}$ & 1-tensor & $B$ \\
\hline$R_{j}(f)$ & $\nabla\left[\frac{1}{\rho} \nabla \times\left(\frac{\delta f}{\delta \mathbf{B}^{*}}\right)\right]-\frac{1}{T} \frac{\delta f}{\delta \sigma} \nabla$ & 2 -tensor & $j$ \\
\hline$R_{T 2}(f)$ & $\nabla\left(\frac{1}{\rho} \mathbf{B} \times \nabla\left(\frac{1}{T} \frac{\delta f}{\delta \sigma}\right)\right)$ & 2 -tensor & $T 2$ \\
\hline
\end{tabular}

TABLE 1. The several kinds of thermodynamic responses, including their tensor character, for dissipative extended magnetohydrodynamics.

Using (4.8) for the new affinity, the current viscosity will change into

$$
\begin{aligned}
& \nabla\left(\frac{1}{\rho} \nabla \times\left(\frac{\delta f}{\delta \mathbf{B}^{*}}\right)\right) \longrightarrow \nabla\left[\frac{1}{\rho} \nabla \times\left(\frac{\delta f}{\delta \mathbf{B}^{*}}\right)-\frac{1}{T} \frac{\delta f}{\delta \sigma} \frac{\mathbf{j}}{\rho}+\frac{1}{\rho} \mathbf{B} \times \nabla\left(\frac{1}{T} \frac{\delta f}{\delta \sigma}\right)\right] \\
& =\nabla\left(\frac{1}{\rho} \nabla \times\left(\frac{\delta f}{\delta \mathbf{B}^{*}}\right)\right)-\frac{1}{T} \frac{\delta f}{\delta \sigma} \nabla\left(\frac{\mathbf{j}}{\rho}\right) \\
& -\nabla\left(\frac{1}{T} \frac{\delta f}{\delta \sigma}\right) \otimes \frac{\mathbf{j}}{\rho}+\nabla\left(\frac{1}{\rho} \mathbf{B} \times \nabla\left(\frac{1}{T} \frac{\delta f}{\delta \sigma}\right)\right) .
\end{aligned}
$$

Lastly, the term with the second derivative of the temperature becomes

$$
\nabla\left(\frac{1}{\rho} \mathbf{B} \times \nabla\left(\frac{\delta f}{\delta \varepsilon}\right)\right) \longrightarrow \nabla\left(\frac{1}{\rho} \mathbf{B} \times \nabla\left(\frac{1}{T} \frac{\delta f}{\delta \sigma}\right)\right)
$$

From our knowledge of the form of the brackets in the thermodynamic variables and the determination of the affinities in the dynamical coordinates $\left(\sigma, \rho, \mathbf{m}, \mathbf{B}^{*}\right)$, we can re-write the general bracket. In particular, upon replacing the densities of the general bracket of (3.3) by expressions (4.6) - (4.10) above, a bracket with many terms is generated. For efficiency, we will directly develop the temperature terms from the different affinities and add a $T$ factor when it makes things easier. We denote by $\widehat{L}$ the phenomenological tensor in these new coordinates, with indices denoting the processes as explained in table 1 while, as before, we suppress tensorial indices that should be clear from context. Thus, the bracket has the following form:

$$
(f, g)=\int_{\Omega} \frac{T}{\mathcal{T}} R_{\alpha}(f) \widehat{L}_{\alpha \beta} R_{\beta}(g),
$$

where $\alpha$ and $\beta$ are summed over the process index set $\{T, v, B, j, T 2\}$ and the responses $R_{\alpha}$ are explicitly given in table 1. These responses may be 1 - or 2-tensors and are contracted with the phenomenological tensors that are of appropriate rank; e.g., if $R_{\alpha}$ is a $a$-tensor and $R_{\beta}$ is a $b$-tensor, then $\widehat{L}_{\alpha \beta}$ is a $(a+b)$-tensor.

Now, it only remains to find the phenomenological coefficients. For this, we write the various equations of XMHD and identify the terms. We remark that thanks to the Onsager relations, the phenomenological tensor is symmetric and when we determine a 
term we automatically know its dual term. We could use this to shorten calculations, but for completeness we will write out all the terms and discover these symmetries. Proceeding, we see the momentum equation gets the dissipative term

$$
\begin{aligned}
(\mathbf{m}, \mathcal{S}) \mathcal{T} & =\nabla \cdot\left[\frac{1}{T} \widehat{L}_{v T} \nabla T+\widehat{L}_{v v} \nabla \mathbf{v}+\widehat{L}_{v v} \mathbf{j}+\widehat{L}_{v j} \nabla\left(\frac{\mathbf{j}}{\rho}\right)+\widehat{L}_{v T 2} \nabla\left(\frac{1}{\rho T} \mathbf{B} \times \nabla T\right)\right] \\
& =\nabla \cdot\left[\Lambda_{v v} \nabla \mathbf{v}+\Lambda_{v j} \nabla\left(\frac{\mathbf{j}}{\rho}\right)\right] .
\end{aligned}
$$

Thus, we identify the nonvanishing phenomenological coefficients $\widehat{L}_{v v}=\Lambda_{v v}$ and $\widehat{L}_{v j}=$ $\Lambda_{v j}$. Next, for the magnetic field, we get

$$
\begin{aligned}
& \left(\mathbf{B}^{*}, \mathcal{S}\right) \mathcal{T}=-\nabla \times\left[\frac{1}{T} \widehat{L}_{B T} \nabla T+\widehat{L}_{B v} \nabla \mathbf{v}+\widehat{L}_{B B} \mathbf{j}+\widehat{L}_{B j} \nabla\left(\frac{\mathbf{j}}{\rho}\right)\right. \\
& \left.+\widehat{L}_{B T 2} \nabla\left(\frac{1}{\rho T} \mathbf{B} \times \nabla T\right)\right] \\
& +\nabla \times\left(\frac { 1 } { \rho } \nabla \cdot \left[\frac{1}{T} \widehat{L}_{j T} \nabla T+\widehat{L}_{j v} \nabla \mathbf{v}+\widehat{L}_{j B} \mathbf{j}+\widehat{L}_{j j} \nabla\left(\frac{\mathbf{j}}{\rho}\right)\right.\right. \\
& \left.\left.+\widehat{L}_{j T 2} \nabla\left(\frac{1}{\rho T} \mathbf{B} \times \nabla T\right)\right]\right) \\
& =\nabla \times\left[-\left(\eta_{j j} \mathbf{j}+\eta_{j T} \nabla T\right)+\frac{1}{\rho} \nabla \cdot\left(\Lambda_{j v} \nabla \mathbf{v}+\Lambda_{j j} \nabla\left(\frac{\mathbf{j}}{\rho}\right)\right)\right] .
\end{aligned}
$$

Thus, the only nonvanishing coefficients are $\widehat{L}_{B T}=T \eta_{j T}, \widehat{L}_{B B}=\eta_{j j}, \widehat{L}_{j v}=\Lambda_{j v}$, and 
$\widehat{L}_{j j}=\Lambda_{j j}$. Finally, using the previous results, the entropy equation yields

$$
\begin{aligned}
& (\sigma, \mathcal{S}) \mathcal{T}=\nabla\left[\frac{1}{T^{2}} \widehat{L}_{T T} \nabla T+\frac{1}{T} \widehat{L}_{T v} \nabla \mathbf{v}+\frac{1}{T} \widehat{L}_{T B} \mathbf{j}+\frac{1}{T} \widehat{L}_{T j} \nabla\left(\frac{\mathbf{j}}{\rho}\right)\right. \\
& \left.+\frac{1}{T} \widehat{L}_{T T 2} \nabla\left(\frac{1}{\rho T} \mathbf{B} \times \nabla T\right)\right] \\
& +\frac{1}{T^{2}} \nabla T \cdot\left[\frac{1}{T} \widehat{L}_{T T} \nabla T+\widehat{L}_{T v} \nabla \mathbf{v}+\widehat{L}_{T B} \mathbf{j}+\widehat{L}_{T j} \nabla\left(\frac{\mathbf{j}}{\rho}\right)\right. \\
& \left.+\widehat{L}_{T T 2} \nabla\left(\frac{1}{\rho T} \mathbf{B} \times \nabla T\right)\right] \\
& +\frac{1}{T} \nabla v: \Pi_{v}+\frac{1}{T} \nabla\left(\frac{\mathbf{j}}{\rho}\right): \Pi_{v}+\frac{1}{T} \mathbf{j} \cdot \mathbf{J}_{j} \\
& +\frac{1}{T} \nabla \cdot\left(\frac{\mathbf{B}}{\rho} \times \nabla \cdot\left[\frac{1}{T} \widehat{L}_{T 2 T} \nabla T+\widehat{L}_{T 2 v} \nabla \mathbf{v}+\widehat{L}_{T 2 B} \mathbf{j}+\widehat{L}_{T 2 j} \nabla\left(\frac{\mathbf{j}}{\rho}\right)\right.\right. \\
& \left.\left.+\widehat{L}_{T 2 T 2} \nabla\left(\frac{1}{\rho T} \mathbf{B} \times \nabla T\right)\right]\right) \\
& =\nabla \cdot\left(\eta_{T j} \mathbf{j}+\eta_{T T} \nabla T\right)+\frac{1}{T} \nabla T \cdot\left(\eta_{T j} \mathbf{j}+\eta_{T T} \nabla T\right)+\frac{1}{T} \mathbf{j} \cdot \mathbf{J}_{j} \\
& +\frac{1}{T} \nabla \mathbf{v}: \Pi_{v}+\frac{1}{T} \nabla\left(\frac{\mathbf{j}}{\rho}\right): \Pi_{j} .
\end{aligned}
$$

Then, the nonvanishing coefficients are $\widehat{L}_{T T}=T^{2} \eta_{T T}$ and $L_{T B}=T \eta_{T j}$. Observe from the above that indeed the Onsager relations hold.

From the tensor $\widehat{L}$, one could change coordinates back and obtain the tensor $L$. This is an easy computation, but not of our interest here. Similarly, one could have computed the tensor $L$ before changing coordinates to get the tensor $\widehat{L}$. 


\subsection{Metriplectic Framework}

We are now able to write the dissipative part of the metriplectic bracket for XMHD. From our calculations, we have found the following bracket for any functionals $f$ and $g$ :

$$
\begin{aligned}
& (f, g)=\int_{\Omega} \mathrm{d}^{3} y \frac{T}{\mathcal{T}}\left[\nabla ( \frac { 1 } { T } \frac { \delta f } { \delta \sigma ( \mathbf { y } ) } ) \cdot \left\{T^{2} \eta_{T T} \nabla\left(\frac{1}{T} \frac{\delta g}{\delta \sigma(\mathbf{y})}\right)\right.\right. \\
& \left.+T \eta_{T j}\left(\nabla \times\left(\frac{\delta g}{\delta \mathbf{B}^{*}(\mathbf{y})}\right)-\frac{1}{T} \frac{\delta g}{\delta \sigma(\mathbf{y})} \mathbf{j}\right)\right\} \\
& +\left(\nabla \times\left(\frac{\delta f}{\delta \mathbf{B}^{*}(\mathbf{y})}\right)-\frac{1}{T} \frac{\delta f}{\delta \sigma(\mathbf{y})} \mathbf{j}\right) \cdot\left\{T \eta_{j T} \nabla\left(\frac{1}{T} \frac{\delta g}{\delta \sigma(\mathbf{y})}\right)\right. \\
& \left.+\eta_{j j}\left(\nabla \times\left(\frac{\delta g}{\delta \mathbf{B}^{*}(\mathbf{y})}\right)-\frac{1}{T} \frac{\delta g}{\delta \sigma(\mathbf{y})} \mathbf{j}\right)\right\} \\
& +\left(\nabla\left(\frac{\delta f}{\delta \mathbf{m}(\mathbf{y})}\right)-\frac{1}{T} \frac{\delta f}{\delta \sigma(\mathbf{y})} \nabla \mathbf{v}\right):\left\{\Lambda_{v v}\left(\nabla\left(\frac{\delta g}{\delta \mathbf{m}(\mathbf{y})}\right)-\frac{1}{T} \frac{\delta g}{\delta \sigma(\mathbf{y})} \nabla \mathbf{v}\right)\right. \\
& \left.+\Lambda_{v j}\left(\nabla\left(\frac{1}{\rho} \nabla \times \frac{\delta g}{\delta \mathbf{B}^{*}(\mathbf{y})}\right)-\frac{1}{T} \frac{\delta g}{\delta \sigma(\mathbf{y})} \nabla\left(\frac{\mathbf{j}}{\rho}\right)\right)\right\} \\
& +\left(\nabla\left(\frac{1}{\rho} \nabla \times \frac{\delta f}{\delta \mathbf{B}^{*}(\mathbf{y})}\right)-\frac{1}{T} \frac{\delta f}{\delta \sigma(\mathbf{y})} \nabla\left(\frac{\mathbf{j}}{\rho}\right)\right) \\
& :\left\{\Lambda_{j v}\left(\nabla\left(\frac{\delta g}{\delta \mathbf{m}(\mathbf{y})}\right)-\frac{1}{T} \frac{\delta g}{\delta \sigma(\mathbf{y})} \nabla \mathbf{v}\right)\right. \\
& \left.\left.+\Lambda_{j j}\left(\nabla\left(\frac{1}{\rho} \nabla \times \frac{\delta g}{\delta \mathbf{B}^{*}(\mathbf{y})}\right)-\frac{1}{T} \frac{\delta g}{\delta \sigma(\mathbf{y})} \nabla\left(\frac{\mathbf{j}}{\rho}\right)\right)\right\}\right] .
\end{aligned}
$$

When this bracket of (4.11) is subtracted from the Poisson bracket of (1.5) one obtains the complete metriplectic geometrical formulation of dissipative XMHD.

In closing this section let us discuss the forms of the several dissipative tensors. Throughout this work, we have made no hypotheses on the forms of the various tensors nor on their dependencies on any variables or on phenomenological coefficients. Our only requirement was that there be no cross-effects between different tensor-types of responses, a property that comes from space-parity symmetry (de Groot \& Mazur 1984). Yet, physical symmetries will impose other constraints (de Groot \& Mazur 1984; Landau \& Lifshitz 1960). Time-reversal symmetry will give the Onsager relations that we have already evoked but not used. Moreover, Galilean symmetry will constrain the form and dependency of the tensors. Only the magnetic field can provide directional dependence in the tensors. Anisotropy in Hamiltonian magnetofluids can be introduced by adding a $|\mathbf{B}|$ dependence to the internal energy $u$ (Morrison 1982; Kimura \& Morrison 2014). Pairing this with anisotropic dissipation would be an interesting avenue to explore in the future. Without anisotropy, the 2-tensors would reduce to scalars while the 4-tensors would decompose into a symmetrization operator, an antisymmetricization operator, and a trace operator.

Another constraint is the nonnegativity, which assures the second law of thermodynamics. If we decompose the various tensors into several scalars, the nonnegativity constraint leads to nonnegative scalars for direct effects (as distinct from cross effects) and bounds 
on the norm of the cross-effect scalars by the geometric means of the two direct-effect scalars of the same kind.

We have seen that the construction of the brackets does not require physical symmetries, which provides interesting insight; viz., the bracket formalism is more general than the physics at hand. Symmetries only restrict the form of the bracket; there is still freedom to select any dependance of the scalars on the phase space and anisotropy due to magnetic directional dependance.

\section{Dissipation in the Lagrangian Picture - an Example of Metriplectic Reduction}

\subsection{Lagrangian Picture}

So far, the formalisms of this paper, both Hamiltonian and dissipative, have been in terms of the Eulerian (spatial) picture of fluid mechanics. Thus, a natural question to ask is what would our results look like in the Lagrangian picture, where one tracks fluid elements. For the Hamiltonian part, the relationship between the Eulerian and Lagrangian pictures is well understood for neutral fluid mechanics (see e.g., Morrison 1998a, for review), magnetohydrodynamics (Morrison 2009), and XMHD (Keramidas Charidakos et al. 2014). In the Lagrangian picture one has a canonical Poisson bracket, as expected for a particle-like theory, that reduces to a noncanonical Poisson bracket like the one of equation (1.5) in the Eulerian picture. However, the form of dissipation in the Lagrangian picture that reduces to the dissipative bracket is not evident. Indeed, the lions share of out-of-equilibrium thermodynamics is studied within the Eulerian picture.

Let us briefly recall the Lagrangian picture. In this picture one follows a continuum of particles, labeled by $\mathbf{a}$ and then obtains a flow $\varphi(\mathbf{a}, t)$ that gives the position of the particle, a fluid element, labeled by $\mathbf{a} \in \Omega$ at time $t \in \mathbb{R}$. The configuration space is then the space of the diffeomorphisms of the space $\Omega$. Its cotangent space then defines the momentum $\pi$ and the cotangent bundle will be the phase space. In the Hamiltonian setting of the Lagrangian picture, one attaches attributes to a fluid element (see e.g. Morrison (2009)), viz. mass density $\rho_{0}(\mathbf{a})$, entropy density $\sigma_{0}(\mathbf{a})$, and for magnetohydrodynamics, the magnetic field $\mathbf{B}_{0}(\mathbf{a})$. Then, from $\rho_{0}$ and $\sigma_{0}$ we may infer a temperature $T_{0}(\mathbf{a})$. Using the Lagrange to Euler map, the flow is used to obtain the Eulerian velocity field $\mathbf{v}$ and the attributes are transformed into their wellknown Eulerian counterparts that satisfy the usual equations for the ideal fluid and/or magnetohydrodynamics.

When dissipation is included, we no longer expect attributes to remain independent of time. For example, the initial entropy $\sigma_{0}(\mathbf{a}, t)$ obtains time dependence, which is consistent with the Eulerian version of this quantity no longer being conserved in the Eulerian picture. Our goal is to find the Lagrangian equations that determine this time dependence, consistence with our Eulerian metriplectic dynamics.

For XMHD the situation is more complicated. Given that our derivation of section 2 starts from two-fluid theory, we expect there to be two displacement variables. While $\varphi(\mathbf{a}, t)$ will give a center-of-mass displacement, just as for magnetohydrodynamics, we now have $\varphi_{d}(\mathbf{a}, t)$ that will evaluate the difference of positions between of ions and electrons of a same label. More precisely, we define $\varphi_{d}$ as the additional advection of the magnetic field, which will become clearer when we look at the equations of motion. Conjugate to $\varphi_{d}$, we have a momentum variable $\pi_{d}(\mathbf{a}, t)$. 
The Lagrange to Euler map will be given by the following expressions:

$$
\begin{aligned}
\rho(\mathbf{x}, t) & =\int_{\Omega} \mathrm{d}^{3} a \rho_{0}(\mathbf{a}) \delta_{\Omega}(\mathbf{x}-\varphi(\mathbf{a}, t)), \\
\sigma(\mathbf{x}, t) & =\int_{\Omega} \mathrm{d}^{3} a \sigma_{0}(\mathbf{a}, t) \delta_{\Omega}(\mathbf{x}-\varphi(\mathbf{a}, t)), \\
\mathbf{m}(\mathbf{x}, t) & =\int_{\Omega} \mathrm{d}^{3} a \pi(\mathbf{a}, t) \delta_{\Omega}(\mathbf{x}-\varphi(\mathbf{a}, t)),
\end{aligned}
$$

where recall $\delta_{\Omega}$ is the Dirac distribution. Observe, contrary to the usual reduction expressions (e.g., Morrison 1998a, 2009), here the attribute $\sigma_{0}$ has explicit time dependence, but since we are not allowing particle production this is not the case for $\rho_{0}$. The magnetic field is trickier, but if the displacement variation $\varphi_{d}$ is well defined, the magnetic field is advected as a 2 -form by $\varphi+\varphi_{d}$ (D'Avignon et al. 2016), and we have

$$
\mathbf{B}(\mathbf{x}, t)=\int_{\Omega} \mathrm{d}^{3} a\left(\mathrm{~d} \varphi+\mathrm{d} \varphi_{d}\right) \mathbf{B}_{0}(\mathbf{a}, t) \delta_{\Omega}\left(\mathbf{x}-\varphi(\mathbf{a}, t)-\varphi_{d}(\mathbf{a}, t)\right)
$$

and again observe $\mathbf{B}_{0}$ has explicit time dependence.

From the form of the Lagrange to Euler map above, we see that given the set of variables $\left(\varphi, \varphi_{d}, \pi, \pi_{d}\right)$ and known attributes $\left(\rho_{0}, \sigma_{0}, \mathbf{B}_{0}\right)$, the Eulerian variables are uniquely determined. However, because of relabeling symmetry and the split between orbit behavior and attribute dynamics, the inverse is not true; i.e., given the Eulerian variables, the Lagrangian $\left(\varphi, \varphi_{d}, \pi, \pi_{d}\right)$ and attributes $\left(\rho_{0}, \sigma_{0}, \mathbf{B}_{0}\right)$ are not uniquely determined. Consequently, like the usual case for Hamiltonian reduction, the Lagrange to Euler map is a reduction. Our goal is to find expressions in the Lagrangian picture that reduce to the known Eulerian equations of the metriplectic dynamical systems that we have described in this paper. This is an example of metriplectic reduction, an idea that was introduced in Materassi \& Morrison (2018).

Here we will choose a particular section that accomplishes metriplectic reduction, even though it is implicit and has a degree of arbitrariness. In particular, as mentioned above, we will choose a most natural one, where the dissipation changes the attributes, the fluid element labeled properties, and not the dynamical displacements. For example, irreversible processes will make $\sigma_{0}$ depend on time and increase without altering the form of advection.

\subsection{Relations for Change of Variables}

In order to find our brackets in the Lagrangian picture, we must use the functional chain rule. This is done by comparing Lagrangian and Eulerian variations. However, unlike the usual case of Hamiltonian reduction we include attribute variation. As for Hamiltonian reduction, we have the measure $\mathrm{d}^{3} a$ in the Lagrangian picture and $\mathrm{d}^{3} x$ in the Eulerian picture. These two volume forms differ by a factor of the determinant of the flow $\varphi$. Generalizing the method of D'Avignon et al. (2016), a direct calculation now gives links between functional derivatives of a functional $f$ of Eulerian variables and its 
counterpart $\hat{f}$ of Lagrangian variables,

$$
\begin{aligned}
\frac{\delta \hat{f}}{\delta \pi}(\mathbf{a}, t) & =\frac{\delta f}{\delta \mathbf{m}}(\varphi(\mathbf{a}, t), t), \\
\frac{\delta \hat{f}}{\delta \sigma_{0}}(\mathbf{a}, t) & =\frac{\delta f}{\delta \sigma}(\varphi(\mathbf{a}, t), t), \\
\frac{\delta \hat{f}}{\delta \mathbf{B}_{\mathbf{0}}}(\mathbf{a}, t) & =\left(\mathrm{d} \varphi+\mathrm{d} \varphi_{d}\right)^{T} \frac{\delta f}{\delta \mathbf{B}}\left(\left(\varphi+\varphi_{d}\right)(\mathbf{a}, t), t\right),
\end{aligned}
$$

where $A^{T}$ is the transposed operator of $A$. For XMHD, we need the functional derivative with respect to $\mathbf{B}^{*}$ and not $\mathbf{B}$. A change of variable gives

$$
\frac{\delta f}{\delta \mathbf{B}} \longrightarrow \frac{\delta f}{\delta \mathbf{B}^{*}}+\mu \chi^{2} \nabla \times\left(\frac{1}{\rho} \nabla \times \frac{\delta f}{\delta \mathbf{B}^{*}}\right) .
$$

Using the fact that $\mu$ is of order one, we can invert this relation as a perturbative development in $\mu$. Finally, at order one, we get

$$
\begin{aligned}
& \frac{\delta f}{\delta \mathbf{B}^{*}}\left(\varphi(\mathbf{a}, t)+\varphi_{d}(\mathbf{a}, t), t\right) \approx\left(\mathrm{d} \varphi+\mathrm{d} \varphi_{d}\right)^{-1, T} \frac{\delta \hat{f}}{\delta \mathbf{B}_{\mathbf{0}}}(\mathbf{a}, t) \\
&-\mu \chi^{2} \nabla \times\left(\frac{1}{\rho} \nabla \times\left(\mathrm{d} \varphi+\mathrm{d} \varphi_{d}\right)^{-1, T} \frac{\delta \hat{f}}{\delta \mathbf{B}_{\mathbf{0}}}(\mathbf{a}, t)\right),
\end{aligned}
$$

where the gradients are with respect to $\mathbf{x}=\varphi(\mathbf{a}, t)$.

To be able to perform the change of variables in the Eulerian bracket, we have to express some variables in terms of Lagrangian ones. One can see that $T$ will become $T_{0}$ and $\mathbf{v}$ will become $\pi / \rho_{0}$. The Eulerian gradient will transform to the Lagrangian gradient by $\nabla_{x} f(\varphi(\mathbf{a}))=(\mathrm{d} \varphi)^{-1} \nabla_{a} \hat{f}(\mathbf{a})$. Finally, the electric current will become

$$
\begin{aligned}
\mathbf{j}\left(\left(\varphi+\varphi_{d}\right)(\mathbf{a}, t), t\right) & =\nabla_{x} \times \mathbf{B}\left(\left(\varphi+\varphi_{d}\right)(\mathbf{a}, t), t\right) \\
& =\left(\mathrm{d} \varphi+\mathrm{d} \varphi_{d}\right)^{-1} \nabla_{a} \times\left(\mathrm{d} \varphi+\mathrm{d} \varphi_{d}\right)\left(\mathbf{B}_{0}(\mathbf{a}, t)\right) .
\end{aligned}
$$

From these relations, we have all that is needed to unreduce, i.e., express the brackets in terms of the Lagrangian variables.

Upon effecting this procedure, the Hamiltonian part becomes the canonical bracket,

$$
\{f, g\}=\int_{\Omega} \mathrm{d}^{3} a\left(\frac{\delta f}{\delta \varphi} \cdot \frac{\delta g}{\delta \pi}-\frac{\delta g}{\delta \varphi} \cdot \frac{\delta f}{\delta \pi}+\frac{\delta f}{\delta \varphi_{d}} \cdot \frac{\delta g}{\delta \pi_{d}}-\frac{\delta g}{\delta \varphi_{d}} \cdot \frac{\delta f}{\delta \pi_{d}}\right) .
$$

This result is not surprising given the development of D'Avignon et al. (2016), where the Eulerian bracket is derived from this canonical bracket for extended magnetohydrodynamics. Let us now turn to the dissipative part, which we will first work out explicitly for hydrodynamics.

\subsection{Lagrangian Dissipation for Hydrodynamics}

To make things simple, in this subsection we will first deal with hydrodynamics, i.e., we only consider the usual viscosity and heat conductivity, dropping the magnetic part, which is tedious and presents a subtlety that we will address later. Lagrangian metriplectic dynamics was previously explored in Materassi (2015); however, our study here adds the tools needed to address the magnetic part. For hydrodynamics, the change 
of variables is direct and gives

$$
\begin{aligned}
&(f, g)= \int_{\Omega} \mathrm{d}^{3} a \frac{T_{0}}{\mathcal{T}}\left[\nabla\left(\frac{1}{T_{0}} \frac{\delta f}{\delta \sigma_{0}(\mathbf{a})}\right) \cdot T_{0}^{2} \eta_{T_{0} T_{0}} \nabla\left(\frac{1}{T_{0}} \frac{\delta g}{\delta \sigma_{0}(\mathbf{a})}\right)\right. \\
&+\left(\nabla\left(\frac{\delta f}{\delta \pi(\mathbf{a})}\right)-\frac{1}{T_{0}} \frac{\delta f}{\delta \sigma_{0}(\mathbf{a})} \nabla\left(\frac{\pi}{\rho_{0}}\right)\right) \\
&\left.: \Lambda_{\pi \pi}\left(\nabla\left(\frac{\delta g}{\delta \pi(\mathbf{a})}\right)-\frac{1}{T_{0}} \frac{\delta g}{\delta \sigma_{0}(\mathbf{a})} \nabla\left(\frac{\pi}{\rho_{0}}\right)\right)\right]
\end{aligned}
$$

where we have supressed the explicit time dependence of $\pi$ and $\sigma_{0}$. Here $\eta_{T_{0} T_{0}}=$ $\frac{1}{\left|\mathrm{~d} \varphi^{-1}\right|}\left(\mathrm{d} \varphi^{-1}\right)^{T} \kappa_{T T} \mathrm{~d} \varphi^{-1}$ and $\Lambda_{\pi \pi}=\frac{1}{\left|\mathrm{~d} \varphi^{-1}\right|}\left(\mathrm{d} \varphi^{-1}\right)^{T} \Lambda_{v v} \mathrm{~d} \varphi^{-1}$, with $|\mathrm{d} \varphi|$ the determinant of the endomorphism $\mathrm{d} \varphi$ at each point. To be clear, the multiplication here is composition, and for the 4-tensor $\Lambda$, the contraction is with the first index of each pair, the one linked with the gradient. Hence, these tensors change as 2-form densities under the mapping $\varphi$.

Observe, the form of (5.1) is the same as that for the Eulerian picture, so that the equations will remain the same. Yet, the phenomenological tensors change. They will depend on time and reflect the variation of the physical proximity of labels at nearby points. Let us highlight that even with constant scalar phenomenological tensors in the Eulerian picture (a common assumption), in the Lagrangian picture they will become general time-dependent tensors, unless the displacement $\varphi$ only generates orthogonal transformations, which is coherent physically. Here again, one can see the strength of considering geometrical tools like tensors rather that assuming a particular form like a scalar, which does not exploit the full geometrical structure.

Adding the purely magnetic terms, as opposed to cross terms, is also straightforward for magnetohydrodynamics, with or without the Hall term, and for the full XMHD models. Yet, the expressions are complicated, consequently, we will not write them here. However, the cross terms, magnetic with nonmagnetic, bring new ideas that will be explored in subsection 5.4

\subsection{Nonlocality in the Lagrangian Picture}

Consider now the magnetic cross effects. While the other variables change with $\varphi$ or $\varphi+\varphi_{d}$ directly, allowing an easy change of variables, the cross-effect between a magnetic variable and a nonmagnetic variable change into one of the responses ( $c f$. , table 1) with $\varphi$ while the response changes with $\varphi+\varphi_{d}$. Because there is a product between them, changing variables brings complications. Indeed, we will see that this complication breaks locality in the Lagrangian picture. But first, let us take a look at our general bracket once more.

Recall, for a general nonequilibrium system we saw in section 3 that we have a dissipative bracket of equation (3.3). This bracket can be rewritten as follows:

$$
(f, g)=\frac{1}{\mathcal{T}} \int_{\Omega} \mathrm{d}^{3} x \int_{\Omega} \mathrm{d}^{3} y \frac{\delta f}{\delta \zeta_{\alpha}(\mathbf{x})} \mathcal{L}_{\alpha \beta}(\mathbf{x}, \mathbf{y}) \frac{\delta g}{\delta \zeta_{\beta}(\mathbf{y})},
$$

where $\mathcal{L}_{\alpha \beta}(\mathbf{x}, \mathbf{y}):=\nabla_{x} \nabla_{y}\left(L_{\alpha \beta} \delta_{\Omega}(\mathbf{x}-\mathbf{y})\right)$. Note, $L$ could also depend explicitly on space and on any variable of the phase space but, to be concise, we do not exhibit these dependencies. A generalization of the bracket could then be to allow $\mathcal{L}$ to be a general tensor of distributions. So, why do we have such a special form? First, having only gradient factors $\nabla_{x} \nabla_{y}$ in this distribution is roughly the linear response assumption of out-of-equilibrium thermodynamics. Second, assuming that we have such a Dirac distribution means that the interactions between the variations of $f$ and $g$ exist only 
at the same point. Saying it another way, the value $(f, g)(\mathbf{x})$ depends only on the values of $f$ and $g$ in an arbitrarily small neighborhood of $\mathbf{x}$. This assumption amounts to the assumption of the locality of the interactions.

The complication with cross-terms between magnetic and nonmagnetic terms in XMHD arises because different factors in a term do not transform with the same displacement. One is transformed by $\varphi+\varphi_{d}$ while the other is transformed by $\varphi$. Then, what change of variables do we do? Actually, both. The idea is to express the bracket in its more general form, with two integrals, and to change both variables with their associated displacements. For simplicity, we will only show how this works for the thermoelectric effect, which has the following bracket cross term:

$$
(f, g)_{\mathfrak{T}}:=\int_{\Omega} \mathrm{d}^{3} y \frac{T}{\mathcal{T}} \nabla\left(\frac{1}{T} \frac{\delta f}{\delta \sigma(\mathbf{y})}\right) \cdot T \eta_{T j}\left(\nabla \times\left(\frac{\delta g}{\delta \mathbf{B}^{*}(\mathbf{y})}\right)-\frac{1}{T} \frac{\delta g}{\delta \sigma(\mathbf{y})} \mathbf{j}\right) .
$$

The other terms can be treated similarly.

With the two integrals and a change of the left coordinate using $\varphi$ and the right coordinate using $\varphi+\varphi_{d}$, the bracket becomes the following in the Lagrangian picture:

$$
\begin{aligned}
(f, g)_{\mathfrak{T}}= & \int_{\Omega} \mathrm{d}^{3} a \int_{\Omega} \mathrm{d}^{3} b \frac{T_{0}(\mathbf{a}) T_{0}(\mathbf{b})}{\mathcal{T}} \nabla_{a}\left(\frac{1}{T_{0}(\mathbf{a})} \frac{\delta f}{\delta \sigma_{0}(\mathbf{a})}\right) \cdot \eta_{T_{0} j_{0}} \\
& {\left[\nabla_{b} \times\left(\left(\mathrm{d} \varphi+\mathrm{d} \varphi_{d}\right)^{-1, T} \frac{\delta f}{\delta \mathbf{B}_{\mathbf{0}}}(\mathbf{b})\right.\right.} \\
& \left.-\mu \chi^{2} \nabla_{b} \times\left(\frac{1}{\rho}\left(\mathrm{d} \varphi+\mathrm{d} \varphi_{d}\right)^{-1} \nabla_{b} \times\left(\mathrm{d} \varphi+\mathrm{d} \varphi_{d}\right)^{-1, T} \frac{\delta f}{\delta \mathbf{B}_{\mathbf{0}}}(\mathbf{b})\right)\right) \\
& \left.-\frac{1}{T_{0}(\mathbf{b})} \frac{\delta g}{\delta \sigma_{0}(\mathbf{b})} \nabla_{b} \times\left(\mathrm{d} \varphi+\mathrm{d} \varphi_{d}\right)\left(\mathbf{B}_{0}(\mathbf{b})\right)\right]
\end{aligned}
$$

where

$$
\eta_{T_{0} j_{0}}=\left(\mathrm{d} \varphi^{-1}\right)^{T} \kappa_{T j} \mathrm{~d}\left(\varphi+\varphi_{d}\right)^{-1} \frac{\delta_{\Omega}\left(\varphi(\mathbf{a})-\left(\varphi+\varphi_{d}\right)(\mathbf{b})\right)}{\left|\mathrm{d} \varphi^{-1}\right|\left|\mathrm{d}\left(\varphi+\mathrm{d} \varphi_{d}\right)^{-1}\right|} .
$$

The important point to realize here is that if $\varphi_{d}$ does vanish, then the bracket reduces to a bracket of the same form as that for hydrodynamics. But if $\varphi_{d}$ does not vanish, then the locality assumption breaks. How should this be interpreted? Well, $\varphi_{d}$ is roughly the inertia of the electrons. Thus, this nonlocality is saying that ions and electrons located at the same space point will interact (locality in the Eulerian picture) but that these two kinds of particles do not come from the same label, for they do not have the same dynamics (nonlocality in the Lagrangian picture). Thus, for magnetohydrodynamics and even Hall magnetohydrodynamics, where electron inertia is neglected, locality is saved in the Lagrangian picture; in the equations, $\varphi_{d}$ is identically zero. On the other hand, in $\mathrm{XMHD}$, locality is broken in the Lagrangian picture.

It is interesting to see how a more general bracket, which might have appeared useless, appears naturally in a physical system. Studying more precisely the consequences of such a nonlocality would be a useful avenue for future work. This study also sheds light on the physical consequences of electron inertia.

\section{Conclusion}

In this paper, we have derived a conservative yet dissipative form of XMHD from twofluid theory. We have seen that natural dissipation and cross-effects appear, including a 
new current viscosity. We have seen that this current viscosity is small, explaining why it is mostly neglected. Yet, we have explained it physically and described consequences of its associated cross effects.

We have also constructed a general metriplectic framework for any fluid-like nonequilibrium thermodynamic system and presented a systematic way to derive the dissipative brackets. The main new idea was to use conserved thermodynamic variables, which are natural in this context but differ from the usual Hamiltonian variables, which explains why they are not usually used. As an example, we re-discovered and generalized the hydrodynamic bracket of Morrison (1984b) using this new framework.

With the hydrodynamic experience, we derived for the first time the metriplectic bracket for full dissipative XMHD. We also explained the geometric generality of our result, freeing us from any dependence on the phase space variables or the direction of the magnetic field, thereby obtaining more general equations than typically used for this model.

Finally, we used these geometrical tools to study this model in the Lagrangian picture. In this picture, we still have a natural bracket, but two generalizations appear naturally. First, the geometry of the phenomenological tensor becomes general and time-dependent. This allows for the description when scalar phenomenological tensors are no longer a good approximation. Second, the locality assumption can break, and then we must consider a more general form of dissipative bracket. This occurs for XMHD, because the ions and electrons have separate dynamics.

\section{Acknowledgements}

BC would like to acknowledge the hospitality of the Institute for Fusion Studies and the Department of Physics of The University of Texas at Austin. PJM was supported by the U.S. Department of Energy Contract DE-FG05-80ET-53088 and via a Forschungspreis from the Humboldt Foundation. He warmly acknowledge the hospitality of the Numerical Plasma Physics Division of Max Planck IPP, Garching, where a portion of this research was done.

\section{REFERENCES}

Abdelhamid, H. M., Kawazura, Y. \& Yoshida, Z. 2015 Hamiltonian formalism of extended magnetohydrodynamics. J. Phys. A 48, 235502.

Alfven, H. 1950 Cosmical Electrodynamics. Clarenden Press, Oxford.

Bannon, Peter R. 2003 Hamiltonian description of idealized binary geophysical fluids. American Meteorological Society 60, 2809-2819.

Braginskit, S. I. 1965 Transport Processes in a Plasma. Rev. Plasma Phys. 1, 205-311.

Bressan, C., Kraus, M., Morrison, P. J. \& Maj, O. 2018 Relaxation to magnetohydrodynamics equilibria via collision brackets. Journal of Physics: Conf. Series 1125, 012002.

Callen, H. 1960 Thermodynamics. John Wiley and Sons.

D'Avignon, Eric, Morrison, P. J. \& Pegoraro, F. 2015 Action principle for relativistic magnetohydrodynamics. Physical Review 91, 084050.

D'Avignon, Eric C., Morrison, Philip J. \& Lingam, Manasvi 2016 Derivation of the Hall and extended magnetohydrodynamics brackets. Physics of Plasmas 23, 062101.

EDWARDS, BRIAN J. 1998 An analysis of single and double generator thermodynamic formalisms for the macroscopic description of complex fluids. Journal of Non-Equilibrium Thermodynamics 23 (4), 301-333.

Edwards, B. J. \& Beris, A. N. 1991 Non-canonical poisson bracket for nonlinear elasticity with extensions to viscoelasticity. Journal of Physics A 24, 2461-2480. 
Eldred, Christopher \& Gay-Balmaz, François 2018 Single and double generator bracket formulations of geophysical fluids with irreversible processes. Pre-print.

Eldred, Christopher \& Gay-Balmaz, François 2020 Single and double generator bracket formulations of multicomponent fluids with irreversible processes. Journal of Physics A To appear.

Gay-Balmaz, François \& Yoshimura, Hiroaki $2017 a$ A Lagrangian variational formalism for nonequilibrium thermodynamics. part i: Discrete systems. Journal of Geometry and Physics 111, 169-193.

Gay-Balmaz, François \& Yoshimura, Hiroaki $2017 b$ A Lagrangian variational formalism for nonequilibrium thermodynamics. part ii: Continuum systems. Journal of Geometry and Physics 111, 194-212.

Grmela, M. 1984 Particle and bracket formulations of kinetic equations. Contemporary Mathematics 28, 125-132.

Grmela, Miroslav \& Öttinger, Hans Christian $1997 a$ Dynamics and thermodynamics of complex fluids. i. development of a general formalism. Physical Review E 56 (6).

Grmela, Miroslav \& Öttinger, Hans Christian $1997 b$ Dynamics and thermodynamics of complex fluids. ii. illustration of a general formalism. Physical Review E 56.

de Groot, S. R. \& Mazur, P. 1984 Non-Equilibrium Thermodynamics. Dover Publications.

Hagstrom, G. I. \& Morrison, P. J. 2011 On Krein-like theorems for noncanonical Hamiltonian systems with continuous spectra: Application to Vlasov-Poisson. Trans. Theory and Stat. Phys. 39, 466-501.

Kampen, N. G. Van \& Felderhof, B. U. 1967 Theoretical Methods in Plasma Physics. Interscience Publishers.

Kaufman, A. N. 1984 Dissipative hamiltonian systems: A unifying principle. Physics Letters A 100, 419-422.

Kaufman, A. N. \& Morrison, P. J. 1982 Algebraic structure of the plasma quasilinear equations. Phys. Lett. A 88, 405-406.

Keramidas Charidakos, I., Lingam, M., Morrison, P. J., White, R. L. \& Wurm, A. 2014 Action principles for extended magnetohydrodynamic models. Phisics of Plasmas 21, 092118.

Kimura, K. \& Morrison, P. J. 2014 On energy conservation in extended magnetohydrodynamics. Physics of Plasmas 21, 082101.

Kraus, M. \& Hirvijoki, E. 2017 Metriplectic integrators for the Landau collision operator. Phys. Plasmas 24, 102311.

Kraus, M., Kormann, K., Morrison, P. J. \& Sonnendrücker, E. 2017 GemPiC: Geometric ElectroMagnetic Particle-In-Cell Methods. Journal of Plasma Physics 83, 905830401.

Kulsrud, R. M. 1983 MHD Description of Plasma. In Handbook of Plasma Physics: Basic Plasma Physics (ed. A. A. Galeev \& R. N. Sudan), , vol. 1, chap. 1.4, pp. 115-145. Oxford: North-Holland Publishing Company.

Landau, L. D. \& Lifshitz, E. M. 1960 Course of Theoretical Physics: Mechanics. Pergamon Press.

Lingam, Manasvi, Miloshevich, George \& Morrison, Philip J. 2016 Concomitant Hamiltonian and topological structures of extended magnetohydrodynamics. Physics Letters A 380, 2400-2406.

Lingam, M., Morrison, P. J. \& Miloshevich, G. 2015a Remarkable connections between extended magnetohydrodynamics models. Phys. Plasmas 22, 072111.

Lingam, M., Morrison, P. J. \& TAssi, E. $2015 b$ Inertial magnetohydrodynamics. Phys. Lett A 379, 570-576.

LÜSt, Von ReIMAR 1959 Über die Ausbrecitung von Wellen in einem Plasma. Fortschritte der Physics 7, 503-558.

Materassi, M. 2015 Metriplectic algebra for dissipative fluids in Lagrangian formulation. Entropy 17 (3), 1329-1346.

Materassi, Massimo \& Morrison, Philip J. 2018 Metriplectic torque for rotation control of a rigid body. Cybernetics and Physics 7, 78-86.

Materassi, Massimo \& Tassi, Emanuele 2012 Metriplectic framework for dissipative magneto-hydrodynamics. Physica D: Nonlinear Phenomena 241 (6), 729 - 734. 
Morrison, P. J. 1982 Poisson Brackets for Fluids and Plasmas. AIP Conf. Proc. 88, 13-46.

Morrison, P. J. $1984 a$ Bracket formulation for irreversible classical fields. Phys. Lett. A 100, 423-427.

Morrison, P. J. $1984 b$ Some observations regarding brackets and dissipation. Tech. Rep. PAM228. University of California at Berkeley.

Morrison, P. J. 1986 A paradigm for joined Hamiltonian and dissipative systems. Physica D 18, 410-419.

Morrison, P. J. 1998a Hamiltonian description of the ideal fluid. Reviews of Modern Physics 70, 467-521.

Morrison, P. J. $1998 b$ Thoughts on brackets and dissipation: old and new. Journal of Physics Conference Series 169, 012006.

Morrison, P. J. 2009 On Hamiltonian and Action Principle Formulations of Plasma Dynamics. AIP Conf. Proc. 1188, 329-344.

Morrison, P. J. 2017 Structure and structure-preserving algorithms for plasma physics. Phys. Plasmas 24, 055502.

Morrison, P. J. \& Greene, J. M. 1980 Noncanonical Hamiltonian density formulation of hydrodynamics and ideal magnetohydrodynamics. Phys. Rev. Lett. 45, 790-793.

Morrison, P. J. \& VAnneste, J. 2016 Weakly nonlinear dynamics in noncanonical Hamiltonian systems with applications to fluids and plasmas. Ann. Phys. 368, 117-147.

SAlmon, Rick 1983 Practical use of Hamilton's principle. Journal of Fluid Mechanics 132, 431-444. 